\title{
INJECTIVE BANACH SPACES OF CONTINUOUS FUNCTIONS $\left({ }^{\mathbf{1}}\right)$ \\ BY \\ JOHN WOLFE
}

\begin{abstract}
ABSTRACr. A description is given of the compact Hausdorff spaces $S$ such that the Banach space $C(S)$ of continuous functions on $S$ is a $P_{\lambda}$-space for $\lambda<3$ (under the assumption that $S$ satisfies the countable chain condition). The existence of extension operators from $C\left(X^{*} \backslash X\right)$ to $C\left(X^{*}\right)$ is examined under the assumption that $C\left(X^{*}\right)$ is injective where $X^{*}$ is some compactification of a locally compact extremally disconnected Hausdorff space $X$ (if $C(S)$ is injective, $S$ is of this form). Some new examples of injective spaces $C(S)$ are given.
\end{abstract}

1. Introduction and statement of results. A Banach space $X$ is injective if it has the following extension property: every bounded linear operator from a subspace of a Banach space into $X$ can be extended to a bounded linear operator on the whole space. Goodner [19] introduced a family of Banach spaces equivalent to the injective spaces: for any $\lambda \geqslant 1$, a Banach space $X$ is a $P_{\lambda}$-space if, whenever $X$ is isometrically embedded in another Banach space, there is a projection onto the image of $X$ with norm not larger than $\lambda$. A Banach space is injective iff it is a $P_{\lambda}$-space for some $\lambda \geqslant 1$. Goodner [19], Nachbin [29], and Kelley [23] characterized the $P_{1}$-spaces: a Banach space is a $P_{1}$-space iff it is isometrically isomorphic to the space of continuous functions on an extremally disconnected compact Hausdorff space, i.e., a space $T$ such that the closure of any open set in $T$ is open.

A concrete characterization of the injective Banach spaces seems to be an unmanageable problem at the present time. The outstanding conjecture is that a Banach space is injective iff it is isomorphic to a $P_{1}$-space. This paper deals with the more restricted problem of characterizing those compact Hausdorff spaces whose space of continuous functions is injective. The strongest results are obtained for $P_{\lambda}$-spaces for $\lambda<3$.

Injective spaces of continuous functions have previously been investigated

Received by the editors February 18, 1976.

AMS (MOS) subject classifications (1970). Primary 46B05, 46E15; Secondary 46M10.

Key words and phrases. $P_{\lambda}$-space, injective Banach space, space of continuous functions, Gleason map, averaging operator.

(') The author would like to express his warmly felt gratitude to Professor William G. Bade. This work is contained in the author's doctoral dissertation written under his direction and under NSF grant GP-22712 at the University of California at Berkeley. 
in Amir [1], [2], [4], Banilower [7], Cohen, Labbé and Wolfe [10], Isbell and Semadeni [21], and Wolfe [38]. The bibliography contains a partial list of books and papers which include results on injective Banach spaces.

The letters $S$ and $T$ are reserved for compact Hausdorff spaces and $C(T)$ is the Banach space of real-valued continuous functions on $T$ with the supremum norm. If $J$ is a closed subset of $S$, a bounded linear operator $E$ : $C(J) \rightarrow C(S)$ is an extension operator if $\left.E(f)\right|_{J}=f$ for each $f \in C(J)$.

To state our main result we use the terminology on quotient spaces from pp. 96-99 of Kelley [24].

Definition. Let $S$ be a compact Hausdorff space and let $D_{1}, \ldots, D_{n}$ be disjoint closed subsets of $S$ for some $n \geqslant 2$. For each $i=2, \ldots, n$ let $h_{i}$ : $D_{i} \rightarrow D_{i-1}$ be a homeomorphism from $D_{i}$ into. $D_{i-1}$. Assume $h_{2}: D_{2} \rightarrow D_{1}$ is a homeomorphism onto. For each $s \in S$ define $\bar{s} \in\left(S-\cup_{i=2}^{n} D_{i}\right)$ by $\bar{s}=s$ if $s \notin \cup_{i=2}^{n} D_{i}$ and $\bar{s}=h_{2} \circ h_{3} \circ \ldots \circ h_{i}(s)$ if $s \in D_{i}$ for $i=2, \ldots, n$. Let $\mathbb{D}$ be the partition of $S$ determined by the equivalence relation $s_{1} \sim s_{2}$ if $\bar{s}_{1}=\bar{s}_{2}$. A partition of $S$ obtained in this manner will be called an elementary partition of $S$. If there is an extension operator from $C\left(\cup_{i=1}^{n} D_{i}\right)$ to $C(S)$ then $\mathscr{D}$ will be called an elementary partition of $S$ admitting an extension operator and the quotient space $S / D$ will be called an elementary quotient space of $S$.

It is easily verified that any elementary quotient space is a compact Hausdorff space. The following is a visualization of an elementary partition:

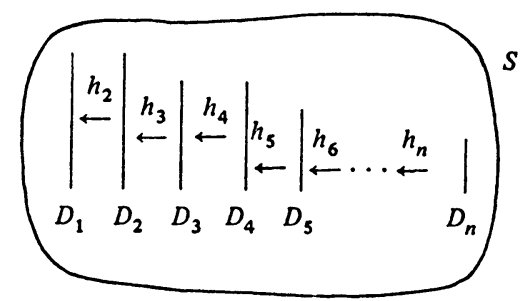

A topological space has the countable chain condition (abbreviated CCC) if every family of disjoint nonempty open sets is countable.

Isbell and Semadeni [21] and Amir [1] showed that if $C(T)$ is $P_{\lambda}$-space for $\lambda<2$ then $T$ is extremally disconnected. In [4], Amir examined $P_{\lambda}$-spaces $C(T)$ for small values of $\lambda$ and obtained strong results for $\lambda<2 \frac{1}{3}$. Our main result follows:

THEOREM 1.1. Suppose $T$ is a compact Hausdorff space satisfying the CCC. If $C(T)$ is a $P_{\lambda}$-space for $\lambda<3$, then $T$ is an elementary quotient space of an extremally disconnected compact Hausdorff space satisfying the CCC.

The following partial converse is elementary (see \$3.1):

Proposition 1.2. If $T$ is an elementary quotient space of an extremally 
disconnected compact Hausdorff space, then $C(T)$ is a $P_{\lambda}$-space for some $\lambda$ (possibly larger than 3).

A continuous function $\phi: S \rightarrow T$ is irreducible if it is onto but no proper closed subset of $S$ is mapped onto $T$. A Gleason map for a space $T$ is an irreducible map $\phi: S \rightarrow T$ where $S$ is extremally disconnected. Every compact Hausdorff space has an (essentially unique) Gleason map (see Gleason [18]).

Isbell and Semadeni [21] have shown that if $C(T)$ is a $P_{\lambda}$-space for $\lambda<3$, then the Gleason map for $T$ is of finite order, i.e., for an integer $n$ the inverse image under the Gleason map for each point in $t$ has cardinality less than $n$.

As an immediate corollary to Theorem 1.1 we get:

Corollary 1.3. Suppose $T$ satisfies the CCC. If $C(T)$ is a $P_{\lambda}$-space for $\lambda<3$, then the Gleason map for $T$ is a local homeomorphism.

Additional technical properties of Gleason maps for spaces $T$ satisfying Corollary 1.3 are contained in Theorem 3.3.1.

THEOREM 1.4. Suppose $C(T)$ is a $P_{\lambda}$-space for $\lambda<3$. Let $U$ be an open set in $T$ and let $A=\bar{U}$ and $M=A \cap(\overline{T \backslash A})$. Then there is an extension operator $E: C(A) \rightarrow C(T)$ (equivalently, there is an extension operator $E: C(M) \rightarrow$ $C(T))$ and so $C(A)$ are $C(M)$ are injective. If $T$ satisfies the $C C C$, then $M$ is extremally disconnected.

$\$ \$ 2$ and 3 contain the proofs of the above results. Proposition 3.1.1 containing a general relationship between averaging (defined below) and extension operators may be of some independent interest.

Any topological space contains a uniquely determined maximal, open, extremally disconnected set since the union of any family of open extremally disconnected sets is open and extremally disconnected. Thus any space $T$ is the union of a maximal open extremally disconnected set and its complementary set which we call the Amir boundary of $T$ and denote by $T_{A}$. Amir [1], [2] proved that if $C(T)$ is injective, then $T_{A}$ is nowhere dense. Thus if $C(T)$ is injective we may view $T$ as a compactification $X^{*}$ of a locally compact extremally disconnected Hausdorff space $X$.

In $\$ 4$ we consider the question of which compactifications $X^{*}$ of locally compact extremally disconnected Hausdorff spaces $X$ have the property that $C\left(X^{*}\right)$ is injective. In particular (Propositions 4.1, 4.2 and 4.3) we show the relationship between the existence of extension operators from the growth $X^{*} \backslash X$ to $X^{*}$ and the existence of extension operators from the growth $\beta(X) \backslash X$ to $\beta(X)(\beta(X)$ is the Stone-Čech compactification of $X)$.

$\$ 5$ contains some new examples of compact Hausdorff spaces $T$ such that $C(T)$ is injective. 
$\$ 6$ contains several remarks, questions and open problems on injective Banach spaces of type $C(T)$.

Let $\phi: S \rightarrow T$ be a map from one compact Hausdorff space onto another. Then $\phi$ determines an isometric embedding $\phi^{\circ}: C(T) \rightarrow C(S)$ of $C(T)$ in $C(S)$ defined by $\phi^{\circ}(f)=f \circ \phi$ for each $f \in C(T)$. A bounded linear operator $u: C(S) \rightarrow C(T)$ is an averaging operator for $\phi$ if $u\left(\phi^{\circ}(f)\right)=f$ for every $f \in C(T)$. The map $\phi$ admits an averaging operator $u$ with $\|u\|<\lambda$ iff there is a projection $P$ of $C(S)$ onto the image $\phi^{\circ}(C(T))$ of $C(T)$ with $\|P\|<\lambda$.

The approach taken in this paper to the injective spaces $C(T)$ is to try to obtain implications from the fact that the Gleason map for $T$ has an averaging operator. For any compact Hausdorff space $T$, the Gleason map $\phi$ : $S \rightarrow T$ provides an isometric embedding $\phi^{\circ}: C(T) \rightarrow C(S)$ of $C(T)$ into the $P_{1}$-space $C(S)$. It follows from this that $C(T)$ is a $P_{\lambda}$-space iff the Gleason map for $T$ has an averaging operator with norm not larger than $\lambda$.

It is hoped that some of the techniques of $\$ 3$ used in studying averaging operators for Gleason maps might prove useful in the analysis of the existence of averaging operators for arbitrary maps between compact Hausdorff spaces.

2. The key proposition and proof of the main theorem. In this section we state the key Proposition 2.1 and prove the main theorem from this proposition. The lengthy proof of the key proposition is contained in the next section.

A set is plural if it contains more than one point.

Key Proposition 2.1. Suppose $T$ is a compact Hausdorff space satisfying the CCC. Suppose $C(T)$ is a $P_{\lambda}$-space for $\lambda<3$ and let $\phi: S \rightarrow T$ be the Gleason map for $T$. Then there are two closed and open disjoint sets $W_{1}$ and $S_{1}$ whose union is $S$ such that

(a) $\left.\phi\right|_{W_{1}}$ is one-to-one and, if $\phi^{-1}(t)$ is plural, then $\phi^{-1}(t) \cap W_{1} \neq \varnothing$,

(b) $D=\left\{\phi^{-1}(t) \cap W_{1}: t \in T\right.$ and $\phi^{-1}(t)$ is plural $\}$ is a closed set,

(c) $\phi_{1}=\left.\phi\right|_{S_{1}}: S_{1} \rightarrow \phi\left(S_{1}\right)$ is a Gleason map having an averaging operator of norm at most $\lambda$.

Remark. To provide some insight into this proposition note that, if $\phi$ was an elementary quotient map and if $W_{1}$ was any closed and open set containing $D_{1}$ and missing $D_{i}$ for $i \geqslant 2$, then parts (a) and (b) of this proposition would be satisfied.

Proof OF THEOREM 1.1. To show that $\phi$ is the quotient map arising from an elementary partition of $S$ we will produce the sets $D_{1}, \ldots, D_{n}$ and into homeomorphisms $h_{2}, \ldots, h_{n}$. Let $W_{1}$ and $S_{1}$ be as in Proposition 2.1. Define $D_{1}=\left\{\phi^{-1}(t) \cap W_{1}: t \in T\right.$ and $\phi^{-1}(t)$ is plural $\}$. Since $\phi_{1}: S_{1} \rightarrow \phi\left(S_{1}\right)$ is a 
Gleason map having an averaging operator with norm less than 3, a reapplication of Proposition 2.1 yields disjoint closed and open sets $W_{2}$ and $S_{2}$ whose union is $S_{1}$ again satisfying (a), (b) and (c). Define $D_{2}=\left\{\phi^{-1}(t) \cap\right.$ $W_{2}: t \in T$ and $\phi^{-1}(t)$ is plural $\}$. To define $h_{2}: D_{2} \rightarrow D_{1}$ note that if $s \in D_{2}$ then, for some $t \in T, s \in \phi^{-1}(t)$ and $\phi^{-1}(t)$ is plural. Thus $\phi^{-1}(t)$ is plural and there is a unique point $s^{\prime}=\left\{\phi^{-1}(t) \cap W_{1}\right\}$ in $D_{1}$. Define $h_{2}(s)=s^{\prime}$. Since $D_{2}$ is closed (and compact) by (b), $h_{2}$ is a homeomorphism. The order of $\phi_{1}$ is one less than the order of $\phi$. We continue applying Proposition 2.1 to $\phi_{i}=\left.\phi\right|_{s_{i}}: S_{i} \rightarrow \phi\left(S_{i}\right)$ for $i>1$ and obtain $W_{i+1}, S_{i+1}, D_{i+1}, h_{i+1}$ and $\phi_{i+1}$. The procedure terminates when we obtain $D_{n}$ where $n$ is the order of $\phi$. Thus $\phi$ is a quotient map arising from an elementary partition.

The existence of an extension operator $E: C\left(\cup_{i=1}^{n} D_{i}\right) \rightarrow C(S)$ follows immediately from Propositions 3.1.2 and 3.1.1(b) which will be proved in the next section. Let $M=\left\{t \in T: \phi^{-1}(t) \cap W_{1} \neq \varnothing\right.$ and $\left.\phi^{-1}(t) \cap S_{1} \neq \varnothing\right\}$. Then by Proposition 3.1.2 there is an extension operator $E: C(M) \rightarrow C(T)$. By (a) of Proposition 2.1, $M=\left\{t \in T: \phi^{-1}(t)\right.$ is plural $\}$ and since $\phi$ is an elementary quotient map, $\phi^{-1}(M)=\cup_{i=1}^{n} D_{i}$. Also $M$ is closed by (b) of Proposition 2.1. Thus by Proposition 3.1.1(b), there is an extension operator from $C\left(\cup_{i=1}^{n} D_{i}\right)$ to $C(S)$ and the proof of Theorem 1.1 is complete.

3. Proof of the Key Proposition. This section contains a lengthy development leading to the proof of the Key Proposition 2.1 at the end. The section is divided into 6 subsections with the first four being independent of one another.

3.1. In this subsection we discuss some relationships between averaging and extension operators. Special cases of the next proposition include Remarks (2) and (3) of Amir [3], Theorem 2.4 of Arens [5], Theorem 1 of Dean [13], Proposition 5.2.3 of Ditor [14] and Proposition 4 of Isbell and Semadeni [21].

Proposition 3.1.1. Let $\phi: S \rightarrow T$ be an onto map. Let $J$ be the closure of the set $\left\{t \in T: \phi^{-1}(t)\right.$ is plural $\}$. Let $J_{1}=\phi^{-1}(J)$ and $\phi_{1}=\left.\phi\right|_{J_{1}}: J_{1} \rightarrow J$.

(a) There is an averaging operator for $\phi \Leftrightarrow$ there is an averaging operator $u_{1}$ for $\phi_{1}$ and a bounded linear operator $E: C\left(J_{1}\right) \rightarrow C(S)$ such that $\left.E(f)\right|_{J_{1}}=f$ for each $f \in C\left(J_{1}\right)$ in the kernel of $u_{1}$.

(b) Suppose there is an averaging operator for $\phi$. Then there is an extension operator $E: C(J) \rightarrow C(T) \Leftrightarrow$ there is an extension operator $E_{1}: C\left(J_{1}\right) \rightarrow$ $C(S)$.

REMARK. This proposition and its proof remain true if $J$ is any closed set containing $\left\{t \in T: \phi^{-1}(t)\right.$ is plural $\}$.

Proof. (a) Suppose $u: C(S) \rightarrow C(T)$ is an averaging operator. Define $u_{1}$ : $C\left(J_{1}\right) \rightarrow C(J)$ for $f \in C\left(J_{1}\right)$ by $u_{1}(f)=\left.u(\tilde{f})\right|_{J}$ where $\tilde{f} \in C(S)$ is any 
extension of $f$. Then $u_{1}$ is well defined since if $\tilde{f}$ and $\tilde{f}^{\prime}$ are two extensions of $f$, then $\tilde{f}-\tilde{f}^{\prime}$ vanishes on $J_{1}$ and $\tilde{f}-\tilde{f}^{\prime}=\phi^{\circ}(h)$ for some $h \in C(T)$ and thus $\left.u\left(\tilde{f}-\tilde{f}^{\prime}\right)\right|_{J}=\left.u\left(\phi^{\circ}(h)\right)\right|_{J}=\left.h\right|_{J}=0$. If $g \in C(J)$ and $\tilde{g} \in C(T)$ is an extension of $g$, then $u_{1}\left(\phi_{1}^{\circ}(g)\right)=\left.u\left(\phi^{\circ}(\tilde{g})\right)\right|_{J}=\left.\tilde{g}\right|_{J}=g$ and thus $u_{1}$ is an averaging operator for $\phi_{1}$. Define $E: C\left(J_{1}\right) \rightarrow C(S)$ for $f \in C\left(J_{1}\right)$ by $E(f)=\tilde{f}-$ $\phi^{\circ} u(\tilde{f})$ where $\tilde{f} \in C(S)$ is any extension of $f$. It is straightforward to verify that $E$ is well defined and has the desired properties. Conversely, if $u_{1}$ and $E$ are given it is straightforward to verify that an averaging operator $u: C(S) \rightarrow$ $C(T)$ is given by $u=\left(\phi^{\circ}\right)^{-1}\left[I-E\left(\phi_{1}^{\circ} u_{1}-I_{1}\right) R\right]$ where $I$ and $I_{1}$ are identity operators on $C(S)$ and $C\left(J_{1}\right)$ and $R: C(S) \rightarrow C\left(J_{1}\right)$ is the restriction operator.

(b) Suppose $u$ is an averaging operator for $\phi$ and let $E_{0}: C(J) \rightarrow C(T)$ be an extension operator. If $E: C\left(J_{1}\right) \rightarrow C(S)$ is as in part (a), then $\phi^{\circ} E_{0} u_{1}+E\left(I-\phi_{1}^{\circ} u_{1}\right): C\left(J_{1}\right) \rightarrow C(S)$ is the desired extension operator. Conversely, if $E_{0}: C\left(J_{1}\right) \rightarrow C(S)$ is an extension operator then so is $u E_{0} \phi_{1}^{\circ}$ : $C(J) \rightarrow C(S)$.

Proof of Proposition 1.2. Suppose that $T$ is an elementary quotient space of the extremally disconnected space $S$ with quotient map $\phi: S \rightarrow T$. Let $D_{1}, \ldots, D_{n}$ and $h_{2}, \ldots, h_{n}$ be as in the definition of elementary quotient space. Then the set $\left\{t \in T: \phi^{-1}(t)\right.$ is plural $\}$ is closed since it equals $\phi\left(D_{1}\right)$. Let $J, J_{1}$ and $\phi_{1}$ be as in the last proposition. Then $J_{1}=\cup_{i=1}^{n} D_{i}$. Define an averaging operator $u_{1}: C\left(J_{1}\right) \rightarrow C(J)$ by $u_{1}(f)=g$ where $g(t)=f\left(\phi^{-1}(t) \cap\right.$ $D_{1}$ ) for $t \in J$. By the definition of elementary quotient space there is an extension operator $E: C\left(J_{1}\right) \rightarrow C(S)$. Thus there is an averaging operator for $\phi$ by (a) of the last proposition and so $C(T)$ is a $P_{\lambda}$-space.

The next proposition generalizes Theorem 2 of Amir [3]. The proof is essentially the same as his.

Proposition 3.1.2. Let $\phi: S \rightarrow T$ be an irreducible onto map, $U$ be a closed and open subset of $S$ and let $M=\left\{t \in T: \phi^{-1}(t) \cap U \neq \varnothing\right.$ and $\phi^{-1}(t) \cap(S$ $\backslash U) \neq \varnothing\}$. If there is an averaging operator for $\phi$ with norm less than 3 then there is an extension operator $E: C(M) \rightarrow C(T)$.

Proof. Let $u: C(S) \rightarrow C(T)$ be an averaging operator for $\phi$ with $\|u\|<3$ $-r, 0<r$. Denote $U$ by $U_{1}$ and let $U_{2}=S \backslash U_{1}$. The characteristic function of $U_{i}$ is denoted $\chi_{U_{i}}$. For $i=1$ or 2 define operators

$$
A_{i}: C(M) \rightarrow C\left(\phi\left(U_{i}\right)\right) \text { for } f \in C(M) \text { by } A_{i}(f)=\left.u\left(\chi\left(s \backslash U_{i}\right) \cdot \phi^{\circ} \tilde{f}\right)\right|_{\phi}\left(U_{i}\right)
$$

where $\tilde{f} \in C(T)$ is any continuous extension of $f$. To see that $A_{i}$ is well defined, let $\tilde{f}$ and $\tilde{f}^{\prime}$ be two extensions of $f$. Then $\tilde{f}-\tilde{f}^{\prime}$ vanishes on $M$ and so $\chi_{\left(s \backslash U_{i}\right)} \cdot \phi^{\circ}\left(\tilde{f}-\tilde{f}^{\prime}\right)=\phi^{\circ}(h)$ where $h \in C(T)$ is defined by $h=0$ and $\phi\left(U_{i}\right)$ 
and $h=\tilde{f}-\tilde{f}^{\prime}$ on $T \backslash \phi\left(U_{i}\right)$. Thus $\left.u\left(\chi_{\left(S \backslash U_{i}\right)} \cdot \phi^{\circ}\left(\tilde{f}-\tilde{f^{\prime}}\right)\right)\right|_{\phi\left(U_{i}\right)}=\left.u\left(\phi^{\circ} h\right)\right|_{\phi\left(U_{i}\right)}$ $=\left.h\right|_{\phi\left(U_{i}\right)}=0$ and $A_{i}$ is well defined.

The restriction maps $R_{i}: C\left(\phi\left(U_{i}\right)\right) \rightarrow C(M)$ are defined by $R_{i}(f)=\left.f\right|_{M}$. We will show that $\left\|R_{i} A_{i}-I\right\|<1$ and thus $R_{i} A_{i}: C(M) \rightarrow C(M)$ is invertible. Then $E_{i}=A_{i}\left(R_{i} A_{i}\right)^{-1}: C(M) \rightarrow C\left(\phi\left(U_{i}\right)\right)$ is an extension operator since $R_{i} E_{i}=1$. Piecing together the halves $E_{1}$ and $E_{2}$ gives the desired extension operator $E: C(M) \rightarrow C(T)$.

We finish the proof by showing $\left\|R_{i} A_{i}-I\right\|<1$. We prove the case $i=2$. Fix $f \in C(M)$ with $\|f\|<1, q \in M, \varepsilon>0$, and an extension $\tilde{f}$ of $f$. Choose an open neighborhood $V$ of $q$ so that, if $t \in V$, then $|f(t)-f(q)|<\varepsilon$ and $\left|u\left(\chi_{U_{1}} \cdot \phi^{\circ} \tilde{f}\right)(t)-u\left(\chi_{U_{1}} \cdot \phi^{\circ} \tilde{f}\right)(q)\right|<\varepsilon$. Since $\phi$ is irreducible, there is an open set $W$ in $T$ with $\phi^{-1}(W) \subset U_{1} \cap \phi^{-1}(V)$. Let $t$ be any point in $W$ and choose $g \in C(T)$ so that $g$ is positive, supported on $W$, and $g(t)=1=\|g\|$. Define $F=(1+f(q)) \phi^{\circ} g-\chi_{U_{1}} \cdot \phi^{\circ} \tilde{f}+\chi_{U_{2}} \phi^{\circ} \tilde{f}$. Then $F \in C(S)$ and $\|F\|<$ $1+\varepsilon$. Since $F=(1+f(q)) \phi^{\circ} g+\phi^{\circ} \tilde{f}-2 \chi_{U_{1}} \phi^{\circ} \tilde{f}, u(F)(t)=1+f(q)+\tilde{f}(t)$ $-2 u\left(\chi_{U_{1}} \phi^{\circ} \tilde{f}\right)(t)=1+f(q)+[\tilde{f}(t)-\tilde{f}(q)]+\tilde{f}(q)-2\left\{\left[u\left(\chi_{U_{1}} \cdot \phi^{\circ} \tilde{f}\right)(t)-\right.\right.$ $\left.u\left(\chi_{U_{1}} \cdot \phi^{\circ} \tilde{f}\right)(q)\right]+u\left(\chi_{U_{1}} \cdot \phi^{\circ} \tilde{f}(q)\right\}>1+2 f(q)-2 A_{2}(f)(q)-3 \varepsilon$. But $(1+$ $\varepsilon)(3-r)>(1+\varepsilon)\|u\| \geqslant u(F)(t)$ and $\varepsilon$ is arbitrary so $A_{2}(f)(q)-f(q) \geqslant$ $-(1-r / 2)$. Since the same inequality holds for $-f,\left|A_{2}(f)(q)-f(q)\right|<1$ $-r / 2$ for $q \in M$ and thus $\left\|R_{2} A_{2}-I\right\| \leqslant 1-r / 2$. This proves the proposition.

REMARK. One can show that if $E: C(M) \rightarrow C(T)$ is the extension operator constructed in the last proposition, then $\|E\| \leqslant(\|u\|-1) /(3-\|u\|)$. This is the inequality obtained by Amir [3].

THEOREM 3.1.3. Suppose $C(T)$ is a $P_{\lambda}$-space for $\lambda<3$ and $T$ satisfies the CCC. Then every boundary for the Gleason map $\phi: S \rightarrow T$ for $T$ is extremally disconnected, i.e. if $U$ is a closed and open subset of $S$, then $M_{U}=\{t \in T$ : $\phi^{-1}(t) \cap U \neq \varnothing$ and $\left.\phi^{-1}(t) \cap(S \backslash U) \neq \varnothing\right\}$ is an extremally disconnected set.

Proof. By the last proposition, if $M$ is a boundary then there is an extension operator $E: C(M) \rightarrow C(T)$. By Lemma 2.5 of Wolfe [38] or p. 230 of Rosenthal [34], $M$ satisfies the CCC. The set $M$ is contained in $P_{\phi}=\{t \in$ $T: \phi^{-1}(t)$ is plural $\}$ and by Lemma 2.3 and Corollary 1.2 of Wolfe [38] the set $\bar{P}$ is extremally disconnected. A topological space is an $F$-space or quasidisconnected if disjoint open $F_{\sigma}$ subsets have disjoint closures. Then $\bar{P}$ is an $F$-space. A closed subset of an $F$-space is an $F$-space by Proposition 24.2.5 of Semadeni [35] so that $M$ is an $F$-space. The theorem now follows from a result of Rosenthal [34, p. 19]: if $X$ is a compact $F$-space satisfying the CCC then $X$ is extremally disconnected.

Proof of TheOREM 1.4. This theorem is an immediate application of the last two results. 
The next proposition will be used in proving part (c) of the Key Proposition. Results on a situation similar to the one covered by this proposition can be found in Proposition 4.6 of Pelkzynski [30] and Proposition 2.8 of Ditor [15].

Proposition 3.1.4. Let $\phi: S \rightarrow T$ be an onto map which has an averaging operator u: $C(S) \rightarrow C(T)$. Suppose $S$ is the disjoint union of closed and open sets $W_{1}$ and $S_{1}$. Let $K=\phi\left(W_{1}\right) \cap \phi\left(S_{1}\right), K_{1}=\phi^{-1}(K) \cap S_{1}, \psi=\left.\phi\right|_{K_{1}}: K_{1} \rightarrow$ $K$, and $\phi_{1}=\left.\phi\right|_{S_{1}}: S_{1} \rightarrow \phi\left(S_{1}\right)$. Then, if $v$ is an averaging operator for $\psi$, then there is an averaging operator $u_{1}$ for $\phi_{1}$ with $\left\|u_{1}\right\|<\|u\|\|v\|$.

Proof. For $f \in C\left(S_{1}\right)$, define $\tilde{f} \in C(S)$ by $\tilde{f}=f$ on $S_{1}$ and $\tilde{f}=\phi_{2}^{\circ}\left(f^{\prime}\right)$ on $W_{1}$ where $\phi_{2}=\left.\phi\right|_{W_{1}}: W_{1} \rightarrow \phi\left(W_{1}\right)$ and where $f^{\prime} \in C\left(\phi\left(W_{1}\right)\right)$ is any extension of $v\left(\left.f\right|_{K_{1}}\right) \in C(K)$ with $\left\|f^{\prime}\right\|=\left\|v\left(\left.f\right|_{K_{1}}\right)\right\|$. Define $u_{1}(f)=u\left(\left.\tilde{f}\right|_{\phi\left(S_{1}\right)}\right.$. Then $u_{1}$ is well defined since, if $f_{1}^{\prime}$ and $f_{2}^{\prime}$ are two extensions of $v\left(\left.f\right|_{K_{1}}\right)$, then $\tilde{f}_{1}-\tilde{f}_{2}=$ $\phi^{\circ}(h)$ where $h=0$ on $\phi\left(S_{1}\right)$ and $h=f_{1}^{\prime}-f_{2}^{\prime}$ on $\phi\left(W_{1}\right)$ and thus $u\left(\tilde{f}_{1}-\tilde{f}_{2}\right)=$ $u\left(\phi^{\circ}(h)\right)=h$ vanishes on $\phi\left(S_{1}\right)$. Furthermore, $u_{1}$ is an averaging operator since, if $f=\phi_{1}^{\circ}(h)$ for $h \in C\left(\phi\left(S_{1}\right)\right)$, we may choose an extension $\tilde{h} \in C(T)$ of $h$ and let $f^{\prime}=\left.\tilde{h}\right|_{\phi\left(W_{1}\right)}$ and then $u_{1}\left(\phi_{1}^{\circ}(h)\right)=\left.u\left(\phi^{\circ}(\tilde{h})\right)\right|_{\phi\left(S_{1}\right)}=h$. Also, $\left\|u_{1}(f)\right\|$ $<\|u\|\|\tilde{f}\|<\|u\|\|v\|\|\tilde{f}\|$ and $\left\|u_{1}\right\|<\|u\|\|v\|$.

3.2. The purpose of this subsection is to state and prove Proposition 3.2.1. We begin by defining the basic concepts of a boundary for an onto map and an $r$-weighted open set for a closed set.

Definition. Let $\phi: S \rightarrow T$ be an onto map. If $U$ is a closed and open subset of $S$ then the set $M_{U}=\left\{t \in T: \phi^{-1}(t) \cap U \neq \varnothing\right.$ and $\phi^{-1}(t) \cap(S \backslash U) \neq$ $\varnothing\}$ is called a boundary for $\phi$.

A simple topological argument shows that if $\phi: S \rightarrow T$ is a Gleason map then $M$ is a boundary for $\phi \Leftrightarrow M$ is a "topological boundary", i.e., there is an open set $W$ such that $M=\bar{W} \cap \overline{(T \backslash \bar{W})}$.

Let $u$ : $C(S) \rightarrow C(T)$ be a bounded linear operator. For a compact Hausdorff space $S, M(S)$ denotes the dual space of $C(S)$ which we identify with the space of regular Borel measures on $S$. For $\mu \in M(S),|\mu|$ is the total variation measure of $\mu$ and $\|\mu\|=|\mu|(S)$. For $t \in T, \delta_{t} \in M(T)$ is defined by $\delta_{t}(f)=f(t)$. The integral representation for $u$ is the map $t \rightarrow \mu_{t}$ of $T$ into $M(S)$ defined by $\mu_{t}=u^{*}\left(\delta_{t}\right)$. The map $t \rightarrow \mu_{t}$ is $w^{*}$ continuous and $\|u\|=$ $\sup \left\{\left\|\mu_{t}\right\|: t \in T\right\}$ (see Proposition 4.1 of Pelczynski [30].) If $\phi: S \rightarrow T$ is an onto map, then $u: C(S) \rightarrow C(T)$ is an averaging operator for $\phi \Leftrightarrow$ for each $t \in T$ and Borel set $B \subset T, \mu_{t}\left(\phi^{-1}(B)\right)=\delta_{t}(B)$ (see Lemma 2.1 of Ditor [15].)

DefinITION. Let $\phi: S \rightarrow T$ be an onto map which has an averaging operator $u$ with integral representation $t \rightarrow \mu_{t}$. Let $M$ be a closed set in $T, G$ an open 
set in $T$ and $r>0$. Then $G$ is an $r$-weighted open set for $M$ (with respect to $u$ ) if $\left|\mu_{t}\right|\left(\phi^{-1}(M)\right)>r$ for each $t \in G$.

Proposition 3.2.1. Let $\phi: S \rightarrow T$ be an irreducible onto map which has an averaging operator $u$ with $\|u\|<3-r$ where $0<r<1$. Let $U$ be a closed and open set in $S$ and let $M_{U}$ be the boundary for $\phi$ determined by $U$. Then there is an open neighborhood $G$ of $M_{U}$ such that $G$ is an r-weighted open set for $M_{U}$.

Proof. For the closed and open set $U$, let $M$ denote the boundary $M_{U}$ and let $U_{1}=U$ and $U_{2}=S \backslash U$. Choose an $\varepsilon>0$ so that $\|u\|<3-(r+\varepsilon)$. Let $t \rightarrow \mu_{t}$ be the integral representation of $u$. For each $q \in M$ choose an open neighborhood $G_{q}$ such that, for $y$ and $z$ in $G_{q},\left|\mu_{y}\left(U_{i}\right)-\mu_{z}\left(U_{i}\right)\right|<\varepsilon / 2$ for $i=1$, 2. Then $G=\cup_{q \in M} G_{q}$ is the desired set. For $t \in M,\left|\mu_{t}\right|\left(\phi^{-1}(M)\right)>$ $\mu_{t}\left(\phi^{-1}(M)\right)=\delta_{t}(M)=1>r$. Thus assume $y \in G_{q}$ for some $q \in M$ and $y \notin M$. We must show that $\left|\mu_{y}\right|\left(\phi^{-1}(M)\right)>r$. Let $M_{i}=\phi^{-1}(M) \cap U_{i}$ for $i=1,2$. Then $0=\delta_{y}(M)=\mu_{y}\left(\phi^{-1}(M)\right)=\mu_{y}\left(M_{1}\right)+\mu_{y}\left(M_{2}\right)$ and so $\left|\mu_{y}\left(M_{1}\right)\right|$ $=\left|\mu_{y}\left(M_{2}\right)\right|$. Since $\phi^{-1}(y)$ is a subset of either $U_{1}$ or $U_{2}$, by symmetry we may suppose $\phi^{-1}(y) \subset U_{1}$. Let $V_{2}=\left\{t: \phi^{-1}(t) \subset U_{2}\right\}$. Then $y \notin V_{2}$ and so $\mu_{y}\left(U_{2}\right)=\mu_{y}\left(M_{2}\right)+\mu_{y}\left(\phi^{-1}\left(V_{2}\right)\right)=\mu_{y}\left(M_{2}\right)+\delta_{y}\left(V_{2}\right)=\mu_{y}\left(M_{2}\right)$. Therefore $\left|\mu_{y}\right|\left(\phi^{-1}(M)\right) \geqslant\left|\mu_{y}\left(M_{1}\right)\right|+\left|\mu_{y}\left(M_{2}\right)\right|=2\left|\mu_{y}\left(M_{2}\right)\right|=2\left|\mu_{y}\left(U_{2}\right)\right|$. Thus it suffices to show that $\left|\mu_{y}\left(U_{2}\right)\right| \geqslant r / 2$.

Suppose to the contrary that $\left|\mu_{y}\left(U_{2}\right)\right|<r / 2$. Since $\phi$ is irreducible, there is a point $z$ with $\phi^{-1}(z) \subset \phi^{-1}\left(G_{q}\right) \cap U_{2}$, i.e., $z \in G_{q}$ and $\phi^{-1}(z) \subset U_{2}$. If $V=\left\{t \in T: \phi^{-1}(t) \subset U_{2}\right\}$, then $z \in V$ and thus $\mu_{z}\left(U_{2}\right)=\mu_{z}\left(M_{2}\right)+$ $\mu_{z}\left(\phi^{-1}(V)\right)=\mu_{z}\left(M_{2}\right)+\delta_{z}(V)=\mu_{z}\left(M_{2}\right)+1$. Thus $\left|\mu_{z}\left(M_{2}\right)\right| \geqslant\left|1-\mu_{z}\left(U_{2}\right)\right|$ $>1-\mu_{y}\left(U_{2}\right)-\left|\mu_{z}\left(U_{2}\right)-u_{y}\left(U_{2}\right)\right| \geqslant 1-(r+\varepsilon) / 2$. As before we have $\left|\mu_{z}\left(M_{1}\right)\right|=\left|\mu_{z}\left(M_{2}\right)\right|$ and thus $\|u\| \geqslant\left\|u_{z}\right\| \geqslant\left|u_{z}\left(\phi^{-1}(z)\right)\right|+\left|u_{z}\left(M_{1}\right)\right|+$ $\left|u_{z}\left(M_{2}\right)\right| \geqslant 1+2\left|u_{z}\left(M_{2}\right)\right|=3-(r+\varepsilon)$. This contradicts the choice of $\varepsilon$ and proves the proposition.

3.3. In this subsection we obtain a topological result (Theorem 3.3.1) about onto maps $\phi: S \rightarrow T$ of finite order where $S$ and $T$ are totally disconnected and every boundary for $\phi$ is extremally disconnected.

We begin with some definitions. Let $\phi: S \rightarrow T$ be an onto map where $S$ and $T$ are compact Hausdorff spaces. A cover $C_{q}=\left\{G ; V_{s}\right\}$ of a finite fiber $Q=\phi^{-1}(q)$ is a closed and open neighborhood $G$ of $q$ together with a disjoint family $\left\{U_{s}\right\}_{s \in Q}$ of open sets in $S$ such that (i) $s \in U_{s}$ for each $s \in Q$ and (ii) $\phi^{-1}(G)=\cup_{s \in Q} U_{s}$. It follows from (ii) that each set $U_{s}$ is closed and open. If $G_{1}$ is a closed and open subset of $G$ with $q \in G_{1} \subset G$, then the refinement $G_{1} \wedge \bigodot_{q}$ of the cover $\bigodot_{q}$ is defined by $G_{1} \wedge \bigodot_{q}=\left\{G_{1} ; U_{s} \cap\right.$ $\left.\phi^{-1}\left(G_{1}\right)\right\}$. The splitting set $M\left(\bigodot_{q}\right)$ of the cover $\bigodot_{q}$ is defined by $M\left(\bigodot_{q}\right)=\{t$ $\in T: Q^{-1}(t) \cap U_{s} \neq \varnothing$ for each $\left.s \in \phi^{-1}(q)\right\}$. Clearly $q \in M\left(\bigodot_{q}\right)$.

These definitions are illustrated by the following example: 


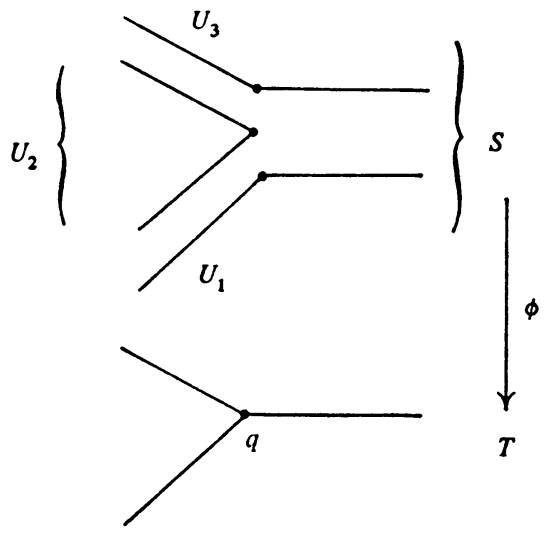

Under the map $\phi$ parallel lines in $S$ are mapped homeomorphically onto the line parallel to them in $T$. Thus the fiber over each point in $T$ is a doubleton except that $\phi^{-1}(q)$ contains 3 points. For the cover $\mathcal{C}_{q}=\left\{T ; U_{1}\right.$, $\left.U_{2}, U_{3}\right\}$, the splitting set $M\left(\mathcal{C}_{q}\right)$ is $\{q\}$. Note that in this example there is no closed and open set $U$ in $S$ such that the boundary $M_{U}$ of $\phi$ determined by $U$ is the same as the splitting set $C_{q}$. For this example, $C_{q}$ has no proper refinement.

The conclusion of the next theorem is in sharp contrast to what happens in the above example. To paraphrase the first part of the theorem, for the setting in which we are interested, each cover of a fiber has a refinement whose splitting set is a boundary.

THEOREM 3.3.1. Let $\phi: S \rightarrow T$ be an onto map of finite order where $S$ and $T$ are totally disconnected compact Hausdorff spaces. Assume that every boundary for $\phi$ is extremally disconnected. Then for any cover $\mathcal{C}_{q}=\left\{G ; U_{s}\right\}$ for a plural fiber $\phi^{-1}(q)$, there is a closed and open set $G_{1}$ with $q \in G_{1} \subset G$ such that

(i) the splitting set for $G_{1} \wedge \mathcal{C}_{q}$ is a boundary for $\phi$, i.e., there is a closed and open set $U$ in $S$ such that $M\left(G_{1} \wedge \mathcal{C}_{q}\right)=\left\{t: \phi^{-1}(t) \cap U \neq \varnothing\right.$ and $\phi^{-1}(t) \cap$ $(S \backslash U) \neq \varnothing\}$.

(ii) There is a point $b \in \phi^{-1}(q)$ such that, if $t \in G_{1}$ and $\left\{s \in \phi^{-1}(q)\right.$ : $\left.\phi^{-1}(t) \cap U_{s} \neq \varnothing\right\}$ is a plural set, then $\phi^{-1}(t) \cap U_{b} \neq \varnothing$.

Two lemmas are needed for the proof of this theorem.

LEMMA 3.3.2. Let $\mathcal{F}$ be a family of nonempty subsets of the finite set $^{-1}(q)$. Assume $\phi^{-1}(q) \in \mathscr{F}$. Suppose that if $F_{1}$ and $F_{2}$ are sets in $\mathscr{F}$; then either $F_{1} \subset F_{2}$ or $F_{2} \subset F_{1}$. Then

(a) there is a point $a \in \phi^{-1}(q)$ such that, if $F \in \mathscr{F}$ and $a \in F$, then $F=\phi^{-1}(q)$.

(b) There is a point $b \in \phi^{-1}(q)$ such that if $F \in \mathcal{F}$ then $b \in F$. 
Proof. The lemma is obvious since we are assuming the sets in $\mathscr{F}$ are linearly ordered. Thus let $a$ be any element of $\phi^{-1}(t) \backslash \cup\{F: F \in \mathcal{F}$ and $\left.F \neq \phi^{-1}(t)\right\}$ and let $b$ be any element of $\cap\{F: F \in \mathcal{F}\}$.

If $\mathcal{C}_{q}=\left\{G ; U_{s}\right\}$ is a cover for the fiber $\phi^{-1}(q)$ and if $t \in G$ then $F\left(t, C_{q}\right)$ denotes a subset of $\phi^{-1}(q)$ defined by $F\left(t, \mathcal{C}_{q}\right)=\left\{s \in \phi^{-1}(q): \phi^{-1}(t) \cap U_{s}\right.$ $\neq \varnothing\}$.

LEMMA 3.3.3. Let $\phi: S \rightarrow T$ be an onto map where $S$ and $T$ are totally disconnected compact Hausdorff spaces. Let $\mathcal{C}_{q}=\left\{G ; U_{s}\right\}$ be a cover of the finite fiber $\phi^{-1}(q)$. Then there is a closed and open set $G_{1} \subset G$ with $q \in G_{1} \subset$ $G$ such that if $t \in G_{1}$ and $F=F\left(t, G_{1} \wedge \bigodot_{q}\right)$ then there is a net $t_{\alpha} \rightarrow q$ such that, for each $t_{\alpha}, F\left(t_{\alpha}, G_{1} \wedge \mathcal{C}_{q}\right)=F$.

Proof. Let $\mathcal{G}=\left\{F: F \subset \phi^{-1}(q)\right.$ and there is a closed and open set $G_{F}$ in $T$ with $q \in G_{F} \subset G$ such that, if $t \in G_{F}$, then $F(t, \mathcal{C}) \neq F$. Define $G_{1}=$ $\cap\left\{G_{F}: F \in \mathcal{G}\right\}$. If $t \in G_{1}$, then $F=F\left(t, G_{1} \wedge \mathcal{C}_{q}\right) \notin \mathcal{G}$. Thus every neighborhood of $q$ has a point $t_{1}$ with $F\left(t_{1}, G_{1} \wedge e_{q}\right)=F$ and the lemma is proved.

Proof of Theorem 3.3.1. Suppose $\mathcal{C}_{q}=\left\{G ; U_{s}\right\}$ is a cover of the fiber $\phi^{-1}(q)$. Let $G_{1}$ be a closed and open set in $T$ with $q \in G_{1} \subset G$ satisying the last lemma. Let $\mathscr{F}=\left\{F \subset \phi^{-1}(q): F=F\left(t, \mathcal{C}_{q}\right)\right.$ for some $t \in G_{1}$ and $F$ is plural $\}$. To show that $\mathscr{F}$ is a linearly ordered family of sets we suppose to the contrary that there are subsets $F_{1}$ and $F_{2}$ in $\mathscr{F}$ and points $p_{1}$ and $p_{2}$ in $\phi^{-1}(q)$ with $p_{1} \in F_{1} \backslash F_{2}$ and $p_{2} \in F_{2} \backslash F_{1}$. Let $U=U_{p_{1}} \cup U_{p_{2}}$. Define open subsets $V_{1}$ and $V_{2}$ of the boundary $M_{U}$ by $t \in V_{i}$ if $t \in M_{U}$ and $\phi^{-1}(t) \cap U_{p_{i}}=\varnothing$. The sets $V_{1}$ and $V_{2}$ are disjoint (since $t \in V_{1} \cap V_{2} \Rightarrow t \notin M_{U}$ ), nonempty (by choice of the $p_{i}$ 's and since the $F_{i}$ 's are plural, $p_{1} \in V_{2}$ and $p_{2} \in V_{1}$ ) and $q \in \bar{V}_{1} \cap \bar{V}_{2}$ (by the last lemma). Thus $M_{U}$ is not extremally disconnected. This proves that the sets in $\mathscr{F}$ satisfy Lemma 3.3.2.

Choose points $a$ and $b$ satisfying (a) and (b) of Lemma 3.3.2. If we let $M$ be the boundary of $\phi$ determined by the closed and open set $U_{a} \cap \phi^{-1}\left(G_{1}\right)$, then $M \subset M\left(G_{1} \wedge \bigodot_{q}\right)$ by (a) $\left(M\left(G_{1} \wedge \bigodot_{q}\right) \subset M\right.$ in general $)$ and so $M=M\left(G_{1}\right.$ $\wedge \mathcal{C}_{q}$ ). This proves part (i) and part (ii) follows from (b) and the definition of F.

3.4. Another ingredient in the proof of the Key Proposition is Theorem 3.4.1 whose proof is the objective of this subsection.

Let $\phi: S \rightarrow T$ be an onto map. If $U$ is an open set in $S$ and $M$ is a closed set in $T$, then $M$ is an $n$-boundary relative to $U$ if there are $n$ disjoint open sets $G_{1}, \ldots, G_{n}$ in $T$ with $\phi^{-1}\left(G_{i}\right) \subset U$ for $i=1, \ldots, n$ and $M=\cap_{i=1}^{n} \bar{G}_{i}$. If $u$ : $C(S) \rightarrow C(T)$ is an averaging operator for $\phi$, then, for $r>0, M$ is an $r$-weight $n$-boundary relative to $U$ if $M$ is an $n$-boundary relative to $U$ and also 
there is an open neighborhood $G$ of $M$ such that $\left|\mu_{t}\right|\left(\phi^{-1}(M)\right)>r$ for each $t \in G$ where $t \rightarrow \mu_{t}$ is the integral representation of $u$.

THEOREM 3.4.1. Let $\phi: S \rightarrow T$ be an onto map and suppose $S$ is extremally disconnected and $u: C(S) \rightarrow C(T)$ is an averaging operator for $\phi$. Let $F$ be a subset of the finite fiber $\phi^{-1}(q)$ with $n=$ cardinality of $F$ and let $r>0$. Then for any $k>1$, there is an open neighborhood $W$ of $F$ such that there is no $r$-weighted $(n+k)$-boundary relative to $W$.

We need a lemma for the proof of this theorem.

LEMMA 3.4.2. Let $\phi: S \rightarrow T$ be an onto map which has an averaging operator $u$. Suppose $S$ is extremally disconnected. Let $\phi^{-1}(q)$ be a finite fiber. Then for any $r>0$ there is a neighborhood $U$ of $q$ such that if $M$ is a closed set in $U$ not containing $q$ and $G$ is any $r$-weighted open set for $M$ then $q \notin \bar{G}$.

Proof. Suppose the conclusion of the lemma is false. Then there is an $r>0$ such that in every neighborhood $U$ of $q$ there is a closed set $M$ and an $r$-weighted open set $G$ for $M$ such that $q \notin M$ and $q \in \bar{G}$.

Claim A. There is a disjoint sequence of closed sets $M_{n}$ and a sequence of open sets $G_{n}$ for $n=1,2, \ldots$ such that for each $n$

(1) $G_{n}$ is an $r$-weighted open set for $M_{n}$,

(2) $q \notin M_{n}$ and

(3) $q \in \bar{G}_{n}$.

The proof of the claim is by induction. As $T$ is a neighborhood of $q$, there is a closed $M_{1}$ and an $r$-weighted open set $G_{1}$ for $M_{1}$ with $q \notin M_{1}$ and $q \in \bar{G}_{1}$. This takes care of the first step. Suppose disjoint closed sets $M_{1}, \ldots, M_{n}$ and open sets $G_{1}, \ldots, G_{n}$ have been chosen satisfying (1), (2), and (3). Since $q \notin M_{i}$ for $i=1, \ldots, n$, there is an open neighborhood $U$ of $q$ which is disjoint from each $M_{i}$ for $i=1, \ldots, n$. So there is a closed set $M_{n+1}$ in $U$ and an $r$-weighted open set $G_{n+1}$ for $M_{n+1}$ with $q \notin M_{n+1}$ and $q \in \bar{G}_{n+1}$. The sets $M_{1}, \ldots, M_{n+1}$ are disjoint since $M_{n+1} \subset U$. This proves the claim.

By assumption, $\phi^{-1}(q)$ has a finite number of points. Suppose $m>$ $\operatorname{card}\left(\phi^{-1}(q)\right)$. Momentarily consider any $m$ open sets $G_{1}, \ldots, G_{m}$ in $T$ such that $q \in \bar{G}_{i}$ for $i=1, \ldots, m$. We assert that there must be distinct integers $i$ and $j$ such that $q \in \overline{G_{i} \cap G_{j}}$. Because if this is not true and if $D$ denotes the set of distinct pairs of integers from 1 to $m$, then for every $(i, j) \in D$ there is an open neighborhood $V_{i j}$ of $q$ such that $V_{i j} \cap\left(G_{i} \cap G_{j}\right)=\varnothing$. Let $V=$ $\cap_{(i, j) \in D} V_{i j}$. Then for any $(i, j) \in D, V \cap\left(G_{i} \cap G_{j}\right)=\varnothing$. But since $V$ is an open neighborhood of $q$ and $q \in \bar{G}_{i}$, it follows that $q \in{\bar{V} \cap G_{i}}_{i}$ for $i=$ $1, \ldots, m$. For $i=1, \ldots, m$ let $W_{i}=V \cap G_{i}$. Then $q \in \bar{W}_{i}$ and the sets $W_{i}$ are now disjoint. Since $S$ is extremally disconnected, the sets $\overline{\phi^{-1}\left(W_{i}\right)}$ are 
disjoint. But $\phi\left(\overline{\phi^{-1}\left(W_{i}\right)}\right)=\bar{W}_{i}$ and $q \in \bar{W}_{i}$, so there is a point in each of the $m$ disjoint sets $\overline{\phi^{-1}\left(W_{i}\right)}$ which is mapped onto $q$. This contradicts the assumption that $m>\operatorname{card}\left(\phi^{-1}(q)\right)$. This proves the assertion that if there are $m$ open sets each one containing $q$ in its closure, then the intersection of some pair of them contains $q$ in its closure.

Using this assertion we now prove the following claim.

Claim B. For every integer $k=1,2, \ldots$, there is a disjoint sequence $M_{1}^{k}$, $M_{2}^{k}, \ldots$ of closed sets and a sequence $G_{1}^{k}, G_{2}^{k}, \ldots$ of open sets such that, for each $n$,

(4) $G_{n}^{k}$ is an $r 2^{(k-1)}$-weighted open set for $M_{n}^{k}$ and

(5) $q \in \bar{G}_{n}^{k}$.

The proof is by induction on $k$. This claim is true for $k=1$ by Claim A. We may assume that there are disjoint sets $M_{1}^{k}, M_{2}^{k}, \ldots$ and open sets $G_{1}^{k}$, $G_{2}^{k}, \ldots$ satisfying (4) and (5). We select the first set $G_{1}^{k+1}$ as an intersection of two of the first $m$ sets $G_{1}^{k}, \ldots, G_{m}^{k}$. By (5), $q \in \bar{G}_{n}^{k}$ for any $n$. Thus by the assertion, there is a pair of distinct integers $i$ and $j$ such that if $G_{1}^{k+1}=G_{i}^{k} \cap$ $G_{j}^{k}$, then $q \in \bar{G}_{1}^{k+1}$. By (4), if $t \in G_{1}^{k+1}$ then $\left|\mu_{t}\right|\left(\phi^{-1}\left(M_{i}^{k}\right)\right) \geqslant r 2^{(k-1)}$ and $\left|\mu_{t}\right|\left(\phi^{-1}\left(M_{j}^{k}\right)\right)>r 2^{(k-1)}$ where $t \rightarrow \mu_{t}$ is the integral representation of $u$. Therefore, since $M_{i}^{k}$ and $M_{j}^{k}$ are disjoint, letting $M_{1}^{k+1}=M_{i}^{k+1} \cup M_{j}^{k+1}$, we get $\left|\mu_{t}\right|\left(\phi^{-1}\left(M_{1}^{k+1}\right)\right) \geqslant 2\left(r 2^{(k-1)}\right)=r 2^{k}$. Therefore, $G_{1}^{k+1}$ is an $\left(r 2^{k}\right)$-weighted open set for $M_{1}^{k+1}$. Thus we have constructed $M_{1}^{k+1}$ and $G_{1}^{k+1}$ from the first "block" of $m$ sets in the sequences $M_{1}^{k}, M_{2}^{k}, \ldots$ and $G_{1}^{k}, G_{2}^{k}, \ldots$ By the same procedure, for any $n, M_{n}^{k+1}$ and $G_{n}^{k+1}$ can be constructed from the $n$th block of $m$ sets in the sequences $M_{1}^{k}, M_{2}^{k}, \ldots$ and $G_{1}^{k}, G_{2}^{k}, \ldots$ This completes the proof of Claim B.

The lemma is now immediate. For any integer $k$, if $t \in G_{n}^{k}$, then, by (4), $\|u\|>\left\|\mu_{\imath}\right\|>\left|\mu_{t}\right|\left(\phi^{-1}\left(M_{n}^{k}\right)\right)>r 2^{(k-1)}$. This contradicts the fact that $\|u\|<$ $\infty$.

Proof of Theorem 3.4.1. Suppose the theorem is false. Then, for some $k>1$, for every neighborhood $W$ of $F$ there is an $r$-weighted $(n+k)$ boundary relative to $W$. We will show that this implies that in every neighborhood $U$ of $q$ there is a closed set $M$ with $q \notin M$ and an $r$-weighted open set $G$ for $M$ with $q \in \bar{G}$. This contradicts the last lemma and proves the theorem.

Let $U$ be any neighborhood of $q$. Let $m=n+k$. Choose a closed and open set $V$ in $S$ with $\phi^{-1}(q) \cap V=F$ and $V \subset \phi^{-1}(U)$. Let $\mathscr{F}$ denote the collection of all $m$-tuples of disjoint open sets $G_{1}, \ldots, G_{m}$ in $T$ such that

(1) $\phi^{-1}\left(G_{i}\right) \subset V$ and

(2) if $M=\cap_{i=1}^{m} \bar{G}_{i}$ then $\cup_{i=1}^{m} G_{i}$ is an $r$-weighted open set for $M$.

To each $m$-tuple $\left\{G_{1}, \ldots, G_{m}\right\}$ in $\mathscr{F}$, let $G=\cup_{i=1}^{m} G_{i}$. Let $\mathscr{F}^{*}$ denote the family of all open sets $G$ arising in this manner. Using Zorn's Lemma we can 
choose a maximal family $\left\{G^{\alpha}\right\}_{\alpha \in I}$ of disjoint members of $\mathscr{F}^{*}$. For each $\alpha \in I$, let $\left\{G_{1}^{\alpha}, \ldots, G_{m}^{\alpha}\right\}$ be an $m$-tuple in $\mathscr{F}$ with $G^{\alpha}=\cup_{i=1}^{m} G_{i}^{\alpha}$.

Define the disjoint open sets $U_{1}, \ldots, U_{m}$ by $U_{i}=\bigcup_{\alpha \in I} G_{i}^{\alpha}$ for $i=$ $1, \ldots, m$. Define the desired open set $G$ by $G=\cup_{i=1}^{m} U_{i}$. Finally, define the desired closed set $M$ by $M=\bigcap_{i=1}^{m} \bar{U}_{i}$.

The proof will be complete when we show that $G$ and $M$ have the properties contradicting Lemma 3.4.2. It is clear that $M$ is a closed subset of $U$. It remains to be shown that $q \notin M$ and $G$ is an $r$-weighted open set for $M$ with $q \in \bar{G}$. To show that $q \notin M$, suppose to the contrary that $q \in M=$ $\cap_{i=1}^{m} \bar{U}_{i}$. Since $\bar{U}_{i}=\overline{\phi\left(\phi^{-1}\left(U_{i}\right)\right)}=\phi\left(\phi^{-1}\left(U_{i}\right)\right), q$ an element of $M$ implies $\phi^{-1}(q) \cap \overline{\phi^{-1}\left(U_{i}\right)} \neq \varnothing$ for each $i=1, \ldots, m$. The sets $U_{1}, \ldots, U_{m}$ are disjoint and, since $S$ is extremally disconnected, the sets $\frac{\phi^{-1}\left(U_{i}\right)}{\phi^{-1}\left(U_{i}\right)}$ are also disjoint. Since $V$ is closed and open, by (3), $\overline{\phi^{-1}\left(U_{i}\right)} \subset V$. Thus we have shown that $\phi^{-1}(q)$ intersects each of the $m$ disjoint subsets $\phi^{-1}\left(U_{1}\right), \ldots, \phi^{-1}\left(U_{m}\right)$ of $V$. Therefore $\operatorname{card}\left(\phi^{-1}(q) \cap V\right) \geqslant m$. However, by the choice of $V, \phi^{-1}(q) \cap V=F$ and $\operatorname{card}(F)=n$. This contradiction proves the $q \notin M$.

To see that $G$ is an $r$-weighted open set for $M$, let $t \in G=\cup_{i=1}^{m} U_{i}=$ $\cup_{i=1}^{m} \cup_{\alpha \in I} G_{i}^{\alpha}$. Then $t \in G_{j}^{\beta}$ for some $j$ and $\beta \in I$. Let $t \rightarrow \mu_{t}$ be the integral representation of the averaging operator $u$. By (2), if $M_{\beta}=\bigcap_{i=1}^{m} \bar{G}_{i}^{\beta}$, then $\left|\mu_{i}\right|\left(\phi^{-1}\left(M_{\beta}\right)\right) \geqslant r$. But $G_{i}^{\beta} \subset U_{i}$ so $M_{\beta}=\bigcap_{i=1}^{m} \bar{G}_{i}^{\beta} \subset \bigcap_{i=1}^{m} \bar{U}_{i}=M$. Therefore $\left|\mu_{l}\right|\left(\phi^{-1}(M)\right) \geqslant\left|\mu_{t}\right|\left(\phi^{-1}\left(M_{\beta}\right)\right)>r$. This proves that $G$ is an $r$ weighted open set for $M$.

Finally, using the maximality of the family $\left\{G_{1}^{\alpha}, \ldots, G_{m}^{\alpha}\right\}_{\alpha \in I}$ we will show that $q \in \bar{G}$. For if $q \notin \bar{G}$ then there is an open neighborhood $W_{0}$ of $q$ which is disjoint from $G$. Let $W=\phi^{-1}\left(W_{0}\right) \cap V$. Then $W$ is a neighborhood of $F$. Therefore, by the assumption that the theorem is false, there is an $r$-weighted $m$-boundary $M_{0}$ relative to $W$. Thus there are disjoint open sets $G_{1}, \ldots, G_{m}$ with $\phi^{-1}\left(G_{i}\right) \subset W \subset V, M_{0}=\cap_{i=1}^{m} \bar{G}_{i}$ and such that $\cup_{i=1}^{m} G_{i}$ is an $r$ weighted open set for $M_{0}$. Thus (1) and (2) are satisfied and so the $m$-tuple $\left(G_{1}, \ldots, G_{m}\right)$ belongs to $\mathscr{F}$. Since $G_{i} \subset W_{0}, G_{i}$ is disjoint from $G=$ $\cup_{i=1}^{m} \cup_{\alpha \in I} G_{i}^{\alpha}$. This contradicts the fact that $\left\{G^{\alpha}\right\}_{\alpha \in I}=\left\{\bigcup_{i=1}^{m} G_{i}^{\alpha}\right\}_{\alpha \in I}$ is a maximal family of disjoint elements of $\mathscr{F}^{*}$. Thus $q \in \bar{G}$. Thus $M$ and $G$ have the desired properties contradicting Lemma 3.4.2.

3.5. In this subsection we prove that the Gleason maps in which we are interested are local homeomorphisms.

An onto map $\phi: S \rightarrow T$ is a local homeomorphism if each point $s \in S$ has a neighborhood $U$ on which $\phi$ is one-to-one. Since $S$ is compact this definition is equivalent to each point in $S$ having a neighborhood mapped homeomorphically onto its image.

THEOREM 3.5.1. Suppose $\phi: S \rightarrow T$ is a Gleason map having an averaging 
operator with norm less than 3. Suppose T satisfies the CCC. Then $\phi$ is a local homeomorphism.

LEMMA 3.5.2. Let $\phi: S \rightarrow T$ be an onto map of finite order. Suppose $\phi$ is not a local homeomorphism. Then there is a subset $F$ of some fiber such that for every open neighborhood $U$ of $F$ there is a point $t \in T$ with $\phi^{-1}(t) \subset U$ and $\operatorname{card}\left(\phi^{-1}(t)\right)>\operatorname{card}(F)$.

Proof. Let $p \in S$ and $q=\phi(p)$ and let $Q=\phi^{-1}(q)$. If the conclusion of the lemma is false, each subset $F$ of $\phi$ has an open neighborhood $U_{F}$ such that if $\phi^{-1}(t) \subset U_{F}$ then $\operatorname{card}\left(\phi^{-1}(t)\right) \leqslant \operatorname{card}(F)$. Let $\left\{U_{s}\right\}_{s \in Q}$ be a disjoint family of open sets with $s \in U_{s}$ and $U_{s} \subset U_{F}$ if $s \in F$ (this is possible since $\phi^{-1}(q)$ is finite). Let $V$ be an open neighborhood of $q$ such that $\phi^{-1}(V) \subset$ $\cup_{s \in Q} U_{s}$. If $U=U_{p} \cap \phi^{-1}(V)$, then it follows from the definitions of things that $\phi$ is one-to-one when restricted to $U$. This proves the lemma.

Proof of TheOREM 3.5.1. If a Gleason map $\phi: S \rightarrow T$ has an averaging operator with norm less than 3 then $\phi$ is of finite order (Theorem 1, Isbell and Semadeni [21]) and $T$ is totally disconnected (Amir [4]; Corollary 1.4 of Wolfe [38]). Assuming $\phi$ is not a local homeomorphism and applying Lemma 3.5.2, let $F$ be a subset of some fiber $\phi^{-1}(q)$ such that for every neighborhood $U$ of $F$ there is a point $t \in T$ with $\phi^{-1}(t) \subset U$ and $\operatorname{card}\left(\phi^{-1}(t)\right)>n$ where $n=\operatorname{card}(F)$. Suppose $\phi$ has an averaging operator with norm smaller than $3-r$ where $0<r$. By Theorem 3.4.1, choose a neighborhood $W$ of $F$ such that there is no $r$-weighted $(n+k)$-boundary relative to $W$ for any $k>1$ with $n+k \leqslant$ order of $\phi$. Choose $t \in T$ with $\phi^{-1}(t) \subset W$ and $\operatorname{card}\left(\phi^{-1}(t)\right) \geqslant$ $n+1$. Choose a disjoint family of open sets $\left\{V_{s}: s \in \phi^{-1}(t)\right\}$ such that $s \in V_{s} \subset W$ for each $s \in \phi^{-1}(t)$. Let $G$ be a closed and open set in $T$ with $t \in G \subset \cup\left\{\phi\left(V_{s}\right): s \in \phi^{-1}(t)\right\}$. If $U_{s}=\phi^{-1}(G) \cap V_{s}$, then $\bigodot_{t}=\left\{G ; U_{s}\right\}$ is a cover for $\phi^{-1}(t)$ with $U_{s} \subset W$ for each $s \in \phi^{-1}(t)$. Since $\phi$ has extremally disconnected boundaries (Theorem 3.1.3), by part (i) of Theorem 3.3.1, there is a closed and open set $G_{0}$ in $T$ with $t \in G_{0} \subset G$ such that if $M=M\left(G_{0} \wedge\right.$ $\left.\mathcal{C}_{t}\right)=\left\{t \in G_{0}: \phi^{-1}(t) \cap U_{s} \neq \varnothing\right.$ for each $\left.s \in \phi^{-1}(t)\right\}$ then $M$ is a boundary relative to $\phi$. Thus $M$ has an $r$-weighted open neighborhood by Proposition 3.2.1. Using the irreducibility of $\phi$ we now obtain the contradiction that $M$ is an $(n+k)$-boundary relative to $W$. For each of the points $s \in \phi^{-1}(t)$ let $G_{s}=\left\{t \in T: \phi^{-1}(t) \subset U_{s} \cap \phi^{-1}\left(G_{0}\right)\right\}$. The sets $G_{s}$ are disjoint and, since $\phi$ is irreducible and $U_{s}$ and $G_{0}$ are closed and open, $\bar{G}_{s}=\phi\left(U_{s}\right) \cap G_{0}$. Thus $\cap\left\{\bar{G}_{s}: s \in \phi^{-1}(t)\right\}=M$ and since $\phi^{-1}\left(G_{s}\right) \subset W$ for each $s$ and card $\left(\phi^{-1}(t)\right)$ $>n, M$ is an $(n+k)$-boundary for some $k \geqslant 1$ contradicting Theorem 3.4.1.

The next lemma follows from the fact that $\phi: S \rightarrow T$ is a closed map since $S$ is compact. 
LEMMA 3.5.3. Suppose $\phi: S \rightarrow T$ is a local homeomorphism. Then $\left\{t: \phi^{-1}(t)\right.$ is plural $\}$ is a closed set in $T$.

3.6. Finally we are in a position to prove the Key Proposition 2.1.

Proof of Proposition 2.1. We begin by constructing a set like $W_{1}$ locally. Let $q \in T$ be any point such that $\phi^{-1}(q)$ is plural. It is known that $T$ is totally disconnected (Wolfe [38, Corollary 1.4], Amir [4]) and that $\phi$ is of finite order (Isbell and Semadeni [21, Theorem 1]). Since $\phi$ is a local homeomorphism (Theorem 3.5.1) we can choose a cover $C_{q}=\left\{G ; U_{s}\right\}$ of the fiber $\phi^{-1}(q)$ such that $\phi$ is one-to-one on each set $U_{s}$. Since each boundary for $\phi$ is extremally disconnected (Theorem 3.1.3), by part (ii) Theorem 3.3.1 there is a closed and open set $G_{q}$ in $T$ with $q \in G_{q} \subset G$ and a point $b \in \phi^{-1}(q)$ such that, if $t \in G_{q}$ and if $\left\{s \in \phi^{-1}(q): \phi^{-1}(t) \cap U_{s}\right\}$ is plural, then $\phi^{-1}(t) \cap U_{b} \neq \varnothing$. Let $W_{q}=\phi^{-1}\left(G_{q}\right) \cap U_{b}$. Thus the set $W_{q}$ satisfies property (a) of the Key Proposition for the restriction map $\left.\phi\right|_{\phi^{-1}\left(G_{q}\right)}$.

Let $K=\left\{t \in T: \phi^{-1}(t)\right.$ is plural $\}$. Since $K$ is compact (Lemma 3.5.3) and totally disconnected we can choose a finite family of pairs $G_{i}$ and $W_{i}$ such that the sets $\left\{G_{i}\right\}$ are a disjoint cover of $K$ by closed and open sets and, for each $i, W_{i}$ satisfies property (a) for the restriction map $\left.\phi\right|_{\phi^{-1}\left(G_{1}\right)}$. Let $W_{1}=$ $\cup W_{i}$. This proves (a). Since $K$ is closed, $D=\phi^{-1}(K) \cap W_{1}$ is also closed. This proves (b). Part (c) follows from Proposition 3.1.4. Note that $K=\phi\left(W_{1}\right)$ $\cap \phi\left(S_{1}\right)$ and so $C(K)$ is a $P_{1}$-space (Theorem 3.1.3) and therefore the map $\psi$ : $K_{1} \rightarrow K$ of Proposition 3.1.4 has an averaging operator $v$ with $\|v\|=1$.

4. Compactifications giving injective spaces of continuous functions and extension operators. Suppose $C(S)$ is an injective Banach space. Then $S$ contains an open dense extremally disconnected subset (Amir [1], [2], Cohen, Labbé and Wolfe [10]). Thus we may view $S$ as a compactification of a locally compact extremally disconnected Hausdorff space.

In this section $X$ will always denote a locally compact extremally disconnected Hausdorff space, $X^{*}$ will denote a compactification of $X, X^{\infty}$ (respectively $\beta(X)$ ) will denote the one-point (respectively Stone-Čech) compactification of $X$. See Gillman and Jerison [17] for a discussion of compactifications.

We consider the question of which compactifications $X^{*}$ ( $X$ as above) have the property that $C\left(X^{*}\right)$ is injective. In particular, we show the relationship between the existence of extension operators from $\beta(X) \backslash X$ to $\beta(X)$ and the existence of extension operators for $X^{*} \backslash X$ to $X^{*}$. The next section contains an example of a space $X^{*}$ such that $C\left(X^{*}\right)$ is injective but there is no extension operator from $X^{*} \backslash X$ to $X^{*}$.

The following results are known: (1) $C\left(X^{\infty}\right)$ is injective $\Leftrightarrow$ there is an extension operator from $\beta(X) \backslash X$ to $\beta(X)$ and (2) $C\left(X^{\infty}\right)$ is a $P_{\lambda}$-space for 
$\lambda<3 \Leftrightarrow \beta(X) \backslash X$ is a finite set (Amir [1, Corollary b]). In this case $\lambda=3-$ $2 / n$ where $n$ is the number of points in $\beta(X) \backslash X$.

The identity map of $X$ to itself uniquely determines an onto map $\rho$ : $\beta(X) \rightarrow X^{*}$. It is easy to show that if $\rho^{-1}(x)$ is plural, then $x \in X^{*} \backslash X$ (see Gillman and Jerison [17, §6.12]). So $\rho$ is the Gleason map for $X^{*}$. Thus if $J=X^{*} \backslash X$, the remark following Proposition 3.1.1 applies and yields the next three propositions.

Proposition 4.1. Let $X^{*}$ be a compactification of the locally compact extremally disconnected Hausdorff space $X$. Suppose there is an extension operator from $X^{*} \backslash X$ to $X^{*}$. Then $C\left(X^{*}\right)$ is injective $\Leftrightarrow C\left(X^{*} \backslash X\right)$ is injective and there is an extension operator from $\beta(X) \backslash X$ to $\beta(X)$.

Proposition 4.2. Let $X^{*}$ be a compactification of the locally compact extremally disconnected Hausdorff space $X$. Suppose that $C\left(X^{*}\right)$ is injective. Then there is an extension operator from $X^{*} \backslash X$ to $X^{*} \Leftrightarrow$ there is an extension operator from $\beta(X) \backslash X$ to $\beta(X)$.

Proposition 4.3. Let $X^{*}$ be a compactification of the locally compact extremally disconnected Hausdorff space $X$. Suppose there is an extension operator from $\beta(X) \backslash X$ to $\beta(X)$. Then $C\left(X^{*}\right)$ is injective $\Leftrightarrow C\left(X^{*} \backslash X\right)$ is injective.

Conway [11, Theorem 2] has shown that if there is an extension operator from $\beta(X) \backslash X$ to $\beta(X)$, then $X$ is pseudocompact i.e., every continuous function on $X$ is bounded. This result plus Proposition 4.2 yield the next corollary.

COROLLARY 4.4. Let $X^{*}$ be a compactification of the locally compact extremally disconnected Hausdorff space $X$. Suppose $C\left(X^{*}\right)$ is injective. If there is an extension operator from $X^{*} \backslash X$ to $X^{*}$, then $X$ is pseudocompact.

5. Examples. This section contains new examples of injective spaces $C(T)$.

The first part outlines a method for constructing such examples. The second part gives an example of an injective space $C(T)$ such that there is no extension operator from $C\left(T_{A}\right)$ to $C(T)$ where $T_{A}$ is the Amir boundary of $T$. All previous examples known to the author have such extension operators.

The structure of these examples is easily seen. For this reason they suggest and illustrate many of the open questions of the next section.

For additional examples of injective spaces $C(T)$, see remark (2) of Amir [3], Amir [1], [4] and Isbell and Semadeni [21].

FIRST EXAMPLES. For each integer $n \geqslant 1$ we construct a space $T_{n}$ such that $C\left(T_{n}\right)$ is injective and such that, if $C\left(T_{n}\right)$ is a $P_{\lambda}$-space, then $\lambda>N$. In certain cases, $C\left(T_{n}\right)$ is a $P_{n}$-space. 
Let $S$ be an extremally disconnected space which contains two disjoint, closed, nowhere dense sets $A_{1}$ and $A_{2}$ which are homeomorphic to $S$. Let $h_{i}$ : $S \rightarrow A_{i}$ be homeomorphisms onto. Suppose there is an extension operator from $A_{i}$ to $S$. If $N$ is the integers then $\beta(N)$ is an example of such a space $S$.

For each integer $n \geqslant 1$, we inductively define an upper semicontinuous closed set partition $D_{n}$ of $S$. Let $D_{1}$ be the trivial partition of $S$, i.e., there are no plural sets in $D_{1}$. The next partition $D_{2}$ is obtained by identifying corresponding points in $A_{1}$ and $A_{2}$. More specifically, using the homeomorphisms $h_{1}: S \rightarrow A_{1}$ and $h_{2}: S \rightarrow A_{2}$, the plural sets of $D_{2}$ are of the form $h_{1}(s) \cup h_{2}(s)$ for $s \in S$. This completes the construction of $D_{2}$. Inductively assume that an upper semicontinuous closed set partition $D_{n-1}$ of $S$ has been defined. Since $S$ is homeomorphic to $A_{1}$ and $A_{2}$, we can place the partition $D_{n-1}$ on each of the sets $A_{1}$ and $A_{2}$ and, in addition, identify corresponding points in $A_{1}$ and $A_{2}$ to obtain a new partition $D_{n}$ of $S$. Thus the plural sets of $D_{n}$ are of the form $h_{1}(F) \cup h_{2}(F)$ where $F \in D_{n-1}$. This completes the construction of the partitions $D_{n}$ of $S$.

For each $n \geqslant 1$, define $T_{n}$ to be the quotient space $S / D_{n}$ and let $\phi_{n}$ : $S \rightarrow T_{n}$ be the quotient map. It is straightforward to verify that $D_{n}$ is upper semicontinuous if $D_{n-1}$ is, and so an inductive proof shows that $T_{n}$ is a compact Hausdorff space.

We state some easily verified facts about the spaces $T_{n}$.

(1) If $K_{2}^{n}=\left\{t \in T_{n}: \phi_{n}^{-1}(t)\right.$ is plural $\}$, then for $n \geqslant 2, \phi_{n}^{-1}\left(K_{2}^{n}\right)=A_{1} \cup A_{2}$ and $K_{2}^{n}=\phi_{n}\left(A_{1} \cup A_{2}\right)=\phi_{n}\left(A_{1}\right)=\phi_{n}\left(A_{2}\right)$ is closed. For $n=1, K_{2}^{n}=\varnothing$.

(2) For $n>1, T_{n}$ is homeomorphic to $K_{2}^{n+1}$ by the natural map making the following diagram commute:

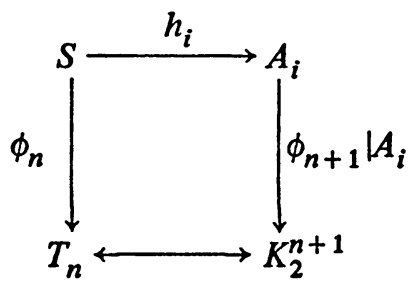

(3) Since $A_{1} \cup A_{2}=\phi_{n}^{-1}\left(K_{2}^{n}\right)$ is nowhere dense, $\phi_{n}$ is irreducible and so $\phi_{n}$ : $S \rightarrow T_{n}$ is the Gleason map for $T_{n}$.

(4) The Amir boundary $\left(T_{n}\right)_{A}$ of $T_{n}$ is homeomorphic to $T_{n-1}$ for $n>2$.

(5) By (1) there is an extension operator from $\phi_{n}^{-1}\left(K_{2}^{n}\right)$ and thus by part (b) of Proposition 3.1.1 there is an extension operator from the Amir boundary $\left(T_{n}\right)_{A}=K_{2}^{n}$ to $T_{n}$ since, as we shall see, there is an averaging operator for $\phi_{n}$.

The proof that $\phi_{n}$ admits an averaging operator is by induction. This is clear for $n=1$ since $\phi_{1}$ is a homeomorphism. Inductively assume there is an 
averaging operator $u_{n}$ for $\phi_{n}$. By (5) there is an extension operator from $\phi_{n+1}^{-1}\left(K_{2}^{n+1}\right)$ to $S$. Therefore, by part (a) of Proposition 3.1.1, there is an averaging operator for $\phi_{n+1}$ if there is an averaging operator $u_{1}$ for $\phi_{n+1}\left|\phi_{n+1}^{-1}\left(K_{2}^{n+1}\right)=\phi_{n+1}\right| A_{1} \cup A_{2}$. The desired averaging operator $u_{1}$ is defined for each $f \in C\left(A_{1} \cup A_{2}\right)$ by $u_{1}(f)=u_{n}\left(f \circ h_{1}\right)$. Thus $C\left(T_{n}\right)$ is injective since $\phi_{n}$ admits an averaging operator.

We give a proof suggested by Haskel Rosenthal that if $S$ satisfies the countable chain condition and $C\left(T_{n}\right)$ is a $P_{\lambda}$-space, then $\lambda>n$. This is obvious if $n=1$. Inductively assume it is true for $n$. If $C\left(T_{n+1}\right)$ is a $P_{\lambda}$-space for $\lambda<n+1$, then, by Theorem 1.1 of Wolfe [38], $C\left(\left(T_{n+1}\right)_{A}\right)$ is a $P_{\lambda-1^{-}}$ space. But by (4), $\left(T_{n+1}\right)=T_{n}$. Thus $C\left(T_{n}\right)$ is a $P_{\lambda-1}$-space where $\lambda-1<(n$ $+1)-1=n$. This contradicts the inductive assumption and proves the result.

It can be proved (without assuming the countable chain condition) that if $C\left(T_{n}\right)$ is a $P_{\lambda}$-space, then $\lambda>n$. We very briefly outline the proof. The proof is based upon the fundamental work on averaging operators by Ditor [15]. For an onto map $\phi: S \rightarrow T$, a certain sequence $\Delta_{\phi}^{(k)}(2, \ldots, 2)$ of subsets of $T$ can be defined (Definition 5.3 of Ditor [15]). If $\Delta_{\phi}^{(k)}(2, \ldots, 2) \neq \varnothing$, then (Corollary 5.4 of Ditor [15]) any averaging operator for $\phi$ has norm at least $k+1$. An argument by induction on $k$ shows that for any $n$ if $F \in D_{n}$ and $\phi_{n}(F) \in \Delta_{\phi_{n}}^{(k)}(2, \ldots, 2)$, then, $\phi_{n+1}\left(h_{1}(F) \cup h_{2}(F)\right) \in \Delta_{n+1}^{(k+1)}(2, \ldots, 2)$. Therefore, since $\Delta_{\phi_{2}}^{(1)}(2, \ldots, 2) \neq \varnothing$ by the irreducibility of $\phi_{2}$, an inductive argument shows that $\Delta_{\phi_{n}}^{(n-1)}(2, \ldots, 2) \neq \varnothing$ for each $n$. Thus if $u_{n}$ is an averaging operator for $\phi_{n}$, then $\left\|u_{n}\right\|>n$. Therefore, if $C\left(T_{n}\right)$ is a $P_{\lambda}$-space, then $\lambda>n$.

If the extension operators from $A_{1}$ and $A_{2}$ to $S$ have norm one, then $C\left(T_{n}\right)$ is a $P_{n}$-space. We briefly outline the proof. Clearly $C\left(T_{1}\right)=C(S)$ is a $P_{1}$-space since $S$ is extremally disconnected. Inductively assume $C\left(T_{n}\right)$ is a $P_{n}$-space. Then there is an averaging operator $u_{n}$ for $\phi_{n}: S \rightarrow T_{n}$ with $\left\|u_{n}\right\|=n$. Let $P_{n}=\phi_{n}^{\circ} \circ u_{n}$ be the projection of $C(S)$ onto $\phi_{n}^{\circ}\left(C\left(T_{n}\right)\right)$. For each $f \in C(S)$, define $A_{n}(f) \in C\left(A_{1} \cup A_{2}\right)$ on each set $A_{i}$ by $A_{n}(f) \mid A_{i}=$ $f \mid A_{i}-\left(P_{n}\left(f \circ h_{i}\right)\right) \circ h_{i}^{-1}$. Since there is a norm one extension operator from each of the disjoint closed sets $A_{1}$ and $A_{2}$, there is an extension operator $E$ : $C\left(A_{1} \cup A_{2}\right) \rightarrow C(S)$ with $\|E\|=1$. Define $P_{n+1}: C(S) \rightarrow C(S)$ for each $f \in C(S)$ by $P_{n+1}(f)=f-E\left(A_{n}(f)\right)$. Then $P_{n+1}$ is a projection of $C(S)$ onto $\phi_{n+1}^{\circ}\left(C\left(T_{n+1}\right)\right)$ and thus $u_{n+1}=\left(\phi_{n+1}^{\circ}\right)^{-1} \circ P_{n+1}$ is an averaging operator for $\phi_{n+1}$. It is immediate that $\left\|A_{n}\right\|=\left\|I-P_{n}\right\|$. It follows from part $\mathrm{c}$ of Lemma 2.2 of Wolfe [38] that if $u$ is an averaging operator for the irreducible onto map $\phi: S \rightarrow T$ and $P=u \circ \phi^{\circ}$, then $\|I-P\|=\|P\|=\|u\|$. Thus $\left\|A_{n}\right\|=\left\|I-P_{n}\right\|=\left\|P_{n}\right\|=\left\|u_{n}\right\|=n$. Therefore, $\left\|u_{n+1}\right\|=\left\|P_{n+1}\right\|=\| I-$ $E \circ A_{n}\|<1+\| E\|\| A_{n} \|=1+n$. This completes the proof that $C\left(T_{n}\right)$ is a 
$P_{n}$-space if the extension operators from $A_{i}$ to $S$ have norm one.

SeCOND EXAMPLE. We construct an injective space $C(T)$ such that there is no extension operator from $C\left(T_{A}\right)$ to $C(T)$ where $T_{A}$ is the Amir boundary of $T$. In fact, $C(T)$ is a $P_{3}$-space.

The author is indebted to H. P. Rosenthal for conversations leading to this example.

Let $\Gamma$ be an uncountable set and $\beta(\Gamma)$ denote the Stone-Čech compactification of $\Gamma$.

Step 1. $\beta(\Gamma)$ is embedded as a nowhere dense subset of an extremally disconnected space $\Omega_{1}$ such that there is a retraction map $\rho: \Omega_{1} \rightarrow \beta(\Gamma)$, i.e., $\rho(x)=x$ for $x \in \beta(\Gamma)$.

Step 2. $\beta(\Gamma)$ is a closed nowhere dense subset of an extremally disconnected space $\Omega_{2}$ such that there is no extension operator from $C(\beta(\Gamma))$ to $C\left(\Omega_{2}\right)$.

Step 3. Let $\Omega$ be the disjoint union of $\Omega_{1}$ and $\Omega_{2}$. Let $T$ be the quotient space of $\Omega$ obtained by identifying corresponding points of $\beta(T)$. Then $T$ is the desired example.

ProOF OF STEP 1. Let $\Omega_{1}=\beta(\Gamma \times N)$ and $\pi: \Gamma \times N \rightarrow \Gamma$ be the projection map. Let $\phi: \beta(\Gamma \times N) \rightarrow \beta(\Gamma)$ be the extension to the Stone-Čech compactifications. Then $\phi(\gamma \times \beta(N))=\gamma$ for each $\gamma \in \Gamma$ and thus $\phi(\beta(\Gamma \times N) \backslash(\Gamma$ $\times N))=\beta(\Gamma)$. A Zorn's lemma argument produces a minimal closed subset $A$ of $\beta(\Gamma \times N) \backslash(\Gamma \times N)$ such that $\phi(A)=\beta(\Gamma)$. Then $\left.\phi\right|_{A}: A \rightarrow \beta(\Gamma)$ is irreducible and thus it is a homeomorphism since $\beta(\Gamma)$ is extremally disconnected (Lemma 2.3, Gleason [18]; Theorem 24.2.10, Semadeni [35]). Note that $A$ is nowhere dense since it is contained in the nowhere dense set $\beta(\Gamma \times N) \backslash$ $(\Gamma \times N)$. Then $\left(\left.\phi\right|_{A}\right)^{-1} \circ \phi: \beta(\Gamma \times N) \rightarrow A$ is the desired retraction map.

Proof of SteP 2. For each cardinal number $m$, let $\Omega_{m}$ denote the maximal ideal space of the Banach algebra $L^{\infty}\left([0,1]^{m}\right)$ (the essentially bounded measurable functions with respect to the product Lebesgue measure on [0, $\left.\left.{ }_{1}\right]^{m}\right)$. Let $\Omega_{2}=\Omega_{m}$ where $m$ is chosen so that the cardinality of $\Omega_{m}$ exceeds the cardinality of $\beta(\Gamma)$ and the weight of $\Omega_{m}$ exceeds the weight of $\beta(\Gamma)$. (The weight of a topological space is the smallest cardinality of a basis for the topology.) Then $\beta(\Gamma)$ embeds as a closed subset of $\Omega_{2}$ (part (e) of Theorem 5.1, Rosenthal [33]). There is no extension operator from $\beta(\Gamma)$ to $\Omega_{2}$ since $\Omega_{2}$ satisfies the CCC but $\beta(\Gamma)$ does not (part (a) of Theorem 4.5, Rosenthal [33]). Finally, $\beta(\Gamma)$ is nowhere dense in $\Omega_{2}$ since any closed and open subset of $\Omega_{2}$ is homeomorphic to $\Omega_{2}\left([0,1]^{m}\right.$ with the product Lebesgue measure is a homogeneous measure space) and thus, by cardinality, $\beta(\Gamma)$ contains no open subset.

Proof of STEP 3. For $\Omega$ and $T$ defined as above, let $\phi: \Omega \rightarrow T$ be the quotient map. By Step 1 and Proposition 3.1.1 (or see remark 3, Amir [3]) 
there is an averaging operator for $\phi$ and thus $C(T)$ is injective (in fact, a $P_{3}$-space). The Amir boundary $T_{A}$ of $T$ is homeomorphic to $\beta(\Gamma)$ (Lemma 2.3, Wolfe [38]) and $\phi^{-1}\left(T_{A}\right)$ is the two disjoint copies of $\beta(\Gamma)$ contained in $\Omega_{1}$ and $\Omega_{2}$. By part (b) of Proposition 3.1.1, there is an extension operator from $C\left(T_{A}\right)$ to $C(T)$ if and only if there is an extension operator from the two disjoint copies of $\beta(\Gamma)$ to $\Omega$. But there is no such extension operator by Step 2.

6. Remarks, questions and open problems. This final section contains a number of questions and open problems about injective spaces of continuous functions. Additional problems can be found in Wolfe [37], [38].

Some of the properties of the spaces $T$ such that $C(T)$ is a $P_{\lambda}$-space for $\lambda<3$ may be true only under the assumption that $C(T)$ is injective. The answers to the following two questions are yes if $C(T)$ is a $P_{\lambda}$-space for $\lambda<3$. They seem to be open for arbitrary injective spaces $C(T)$.

Question 1. If $C(T)$ is injective and $K_{2}=\left\{t \in T: \phi^{-1}(t)\right.$ is plural $\}$ where $\phi: S \rightarrow T$ is the Gleason map for $T$, is $K_{2}$ closed?

Question 2. If $C(T)$ is injective and $U$ is an open subset of $T$, then is $C(\bar{U})$ injective?

The answer to the following two questions is yes if $C(T)$ is a $P_{\lambda}$-space for $\lambda<3$ and $T$ satisfies the CCC.

Question 3. If $C(T)$ is injective, is the Amir boundary $T_{A}$ the boundary of the closure of an open set in $T$ ?

Question 4. If $C(T)$ is injective and if the Gleason map for $T$ is of finite order, is the Gleason map for $T$ a local homeomorphism?

If the answer to question 3 is yes then the answer to question 1 is also yes.

Problem 5. Characterize the compactifications $T$ of discrete spaces such that $C(T)$ is injective.

The answer to the next question is yes if $T$ satisfies the CCC (see Corollary 1.4 of Wolfe [38]).

Question 6. If $C(T)$ is injective, is $T$ totally disconnected?

The answers to all the parts of the following question are yes if $T$ satisfies the CCC.

Question 7. Suppose that $C(T)$ is a $P_{\lambda}$-space for $\lambda<3$ and $T$ does not satisfy the CCC.

(a) Is the Amir boundary of $T$ extremally disconnected?

(b) Is the Gleason map for $T$ a local homeomorphism?

(c) Is the Amir boundary of $T$ the boundary of the closure of an open set in $T ?$

(d) Is there an extension operator from $T_{A}$ to $T$ ?

(e) Is $T$ an elementary quotient space of its Gleason space?

If the answer to (b) or (c) is yes, then so is the answer to (d). 
The techniques in this paper plus a few special arguments yield the following two propositions which are stated without proof. Both are generalizations of Theorem 4 of Amir [4].

Proposition. Suppose $C(T)$ is a $P_{\lambda}$-space for $\lambda<2 \frac{1}{2}$ and $S$ is the Gleason space for $T$. Then there are disjoint, closed, nowhere dense, extremely disconnected subsets $A_{1}, A_{2}, B_{1}, B_{2}, B_{3}$ in $S$ admitting extension operators of norm less than 3 and there are homeomorphisms from $A_{1}$ onto $A_{2}$, from $B_{1}$ onto $B_{2}$ and from $B_{2}$ onto $B_{3}$ such that $T$ is obtained from $S$ by identifying corresponding points in the sets $A_{i}$ and the sets $B_{i}$. Furthermore, the answers to all parts of the last question are yes.

A map $\phi: S \rightarrow T$ is of order exactly $n$ if for every $t \in T$ either $\operatorname{card}\left(\phi^{-1}(t)\right)$ $=n$ or $\operatorname{card}\left(\phi^{-1}(t)\right)=1$.

Proposition. Suppose $C(T)$ is a $P_{\lambda}$-space for $\lambda<3$ and the Gleason map $\phi$ : $S \rightarrow T$ is of order exactly $n$. Then there are $n$ disjoint, closed, nowhere dense, homeomorphic subsets $A_{1}, \ldots, A_{n}$ in $S$ admitting extension operators to $S$ such that $T$ is obtained from $S$ by identifying corresponding points in the sets $A_{i}$. Furthermore, the answers to parts (b) through (e) of the last question are yes (part (a) is open).

Some of the simplest spaces $T$ such that $C(T)$ is injective are those whose Gleason map is of order two. Perhaps a characterization of such spaces would shed light on the more general problem of characterizing those spaces $T$ such that $C(T)$ is injective and the Gleason map for $T$ is of finite order.

Question 8. Suppose $C(T)$ is injective and the Gleason map $\phi: S \rightarrow T$ is of order two. Are there two disjoint, nowhere dense, homeomorphic sets $A_{1}$ and $A_{2}$ in $S$ such that there is an extension operator from $A_{1}$ and such that $T$ is obtained as a quotient of $S$ by identifying pairs of points corresponding via a homeomorphism of $A_{1}$ onto $A_{2}$ ?

A more general version of the last question is this:

Problem 9. Let $T$ be the quotient space of $S$ obtained by identifying corresponding points in two homeomorphic subsets. Let $\phi: S \rightarrow T$ be the quotient map. Give necessary and sufficient conditions for $\phi$ to have an averaging operator.

The techniques of this paper yield the following:

Proposition. If $C(T)$ is injective and the Gleason map for $T$ is of order two, then $C(\bar{U})$ is injective for any open set $U$ in $T$. If $T$ satisfies the countable chain condition then the boundary of the closure of any open set in $T$ is extremally disconnected.

Question 10. Give necessary and sufficient conditions on $S$ in order for $C(S)$ to be a $P_{3}$-space. 
The known examples of spaces $S$ such that $C(S)$ is a $P_{3}$-space are two types: (1) quotient spaces of extremally disconnected spaces obtained by identifying all the points of a nowhere dense closed subset on which there is an extension operator of norm one (Proposition 2 of Isbell and Semadeni [21]) and (2) by taking the Stone-Čech compactification of a disjoint union of a sequence of spaces $S_{n}$ such that $C\left(S_{n}\right)$ is a $P_{\lambda_{n}}$-space where $\lambda_{n}<3$ and $\lambda_{n} \rightarrow 3$ (Lemma 5 of Isbell and Semadeni [21]). A more specific formulation of Question 10 is the following:

Question 11. If $C(S)$ is a $P_{3}$-space, then is $S$ a disjoint union of the above two types?

There is an intimate relationship between averaging and extension operators. This can be seen in Propositions 3.1.1 and 3.1.2. Isbell and Semadeni [21] and Amir [3] have noted that if $\phi: S \rightarrow T$ is a Gleason map, then $\inf \{\|u\|: u$ is an averaging operator for $\phi\}$ is related to the possible values of the infimum of norms of extension operators from closed subsets of compact Hausdorff spaces. A positive resolution of the following three open questions would further the understanding of the spaces $T$ such that $C(T)$ is injective.

Question 12. If $K$ is a closed subset of the extremally disconnected space $S$ and if there is an extension operator from $K$ to $S$, is $K$ extremally disconnected?

Question 13. Does there exist a closed subset of an extremally disconnected space for which there is an extension operator but no extension operator of norm 1? (For contrast with the extremally disconnected case, see Benyamini [8].)

Question 14. (See Rosenthal [34].) Let $K$ be a closed subset of an extremally disconnected space and suppose $C(K)$ is injective. Under what conditions does it follow that $K$ is extremally disconnected?

\section{BIBLIOGRAPHY}

1. D. Amir, Continuous function spaces with the bounded extension property, Bull. Res. Council Israel Sect. F. 10F (1962), 133-138. MR 26 \#592.

2. Projections onto continuous function spaces, Proc. Amer. Math. Soc. 15 (1964), 396-402. MR 29 \#2634; erratum, 30, p. 1205.

3. $\ldots$ On projections and simultaneous extensions, Israel J. Math. 2 (1964), 245-248. MR 31 \#5075.

4. - Continuous function spaces with small projection constants, Proc. Sympos. on Functional Analysis, Hiroshima Univ., 1965.

5. R. F. Arens, Projections on continuous function spaces, Duke Math. J. 32 (1965), 469-478. MR 31 \#6108.

6. J. W. Baker, Some uncomplemented subspaces of $C(X)$ of the type $C(Y)$, Studia Math. 36 (1970), 85-103. MR 43 \# 1113.

7. H. Banilower, Simultaneous extensions and projections in $C(S)$, Thesis.

8. Y. Benyamini, Constants of simultaneous extension of continuous functions, Israel J. Math. 16 (1973), 258-262. MR 49 \#5799. 
9. H. B. Cohen, Injective envelopes of Banach spaces, Bull. Amer. Math. Soc. 70 (1964), 723-726. MR 32 \# 1536.

10. H. B. Cohen, M. A. Labbé and J. Wolfe, Norm reduction of averaging operators, Proc. Amer. Math. Soc. 35 (1972), 519-523. MR 48 \#2741.

11. J. B. Conway, Projections and retractions, Proc. Amer. Math. Soc. 17 (1966), 843-847. MR 33 \#3253.

12. M. M. Day, Normed linear spaces, Springer-Verlag, Berlin, 1958. MR 20 \# 1187.

13. D. W. Dean, Subspaces of $C(H)$ which are direct factors of $C(H)$, Proc. Amer. Math. Soc. 16 (1965), 237-242. MR 30 \#3352.

14. S. Z. Ditor, Linear operators of averaging and extension, Thesis, Univ. of California, Berkeley, 1968.

15. Averaging operators in $C(S)$ and lower semicontinuous sections of continous maps, Trans. Amer. Math. Soc. 175 (1973), 195-208.

16. N. Dunford and J. T. Schwartz, Linear operators. I, General theory, Interscience, New York and London, 1958. MR 22 \#8302.

17. L. Gillman and M. Jerison, Rings of continuous functions, Van Nostrand, Princeton, N.J., 1960. MR 22 \#6994.

18. A. M. Gleason, Projective topological spaces, Illinois J. Math. 2 (1958), 482-489. MR 22 \#12509.

19. D. B. Goodner, Projections in normed linear spaces, Trans. Amer. Math. Soc. 69 (1950), 89-108. MR 12, 266.

20. M. Hasumi, The extension property of complex Banach spaces, Tôhoku Math. J. (2) 10 (1958), 135-142. MR 20 \# 7209.

21. J. R. Isbell and Z. Semadeni, Projection constants and spaces of continuous functions, Trans. Amer. Math. Soc. 107(1963), 38-48. MR 26 \#4169.

22. R. P. Kaufman, A type of extension of Banach spaces, Acta Sci. Math. (Szeged) 27 (1966), 163-166. MR 34 \#4872.

23. J. L. Kelley, Banach spaces with the extension property, Trans. Amer. Math. Soc. 72 (1952), 323-326. MR 13, 659.

24. __ General topology, Van Nostrand, Princeton, N. J., 1955. MR 16, 1136.

25. H. E. Lacey, The isometric theory of classical Banach spaces, Springer-Verlag, New York, 1974.

26. H. E. Lacey and H. B. Cohen, On injective envelopes of Banach spaces, J. Functional Analysis 4 (1969), 11-30. MR 30 \#4644.

27. J. Lindenstrauss, Extension of compact operators, Mem. Amer. Math. Soc. No. 48 (1964). MR 31 \# 3828.

28. J. Lindenstrauss and L. Tzafriri, Classical Banach spaces, Lecture Notes in Math., vol. 338, Springer-Verlag, Berlin, 1973.

29. L. Nachbin, A theorem of Hahn-Banach type for linear transformations, Trans. Amer. Math. Soc. 68 (1950), 28-46. MR 11, 369.

30. A. PeXczynski, Linear extensions, linear averagings, and their applications to linear topological classification of spaces of continuous functions, Dissertationes Math. Rozprawy Mat. 58 (1968). MR 37 \#3335.

31. R. S. Phillips, On linear transformations, Trans. Amer. Math. Soc. 48 (1940), 516-541. MR 2, 318.

32. B. Rodnguez-Salinas Palero, Algunas problemas y teoremas de extension de aplicaciones lineals (Some problems and theorems on the extension of linear mappings), Rev. Acad. Ci. Madrid 65 (1971), 677-704. (Spanish) MR 46 \#4160.

33. H. P. Rosenthal, On injective Banach spaces and the spaces $L^{\infty}(\mu)$ for finite measures $\mu$, Acta. Math. 124 (1970), 205-248. MR 41 \#2370.

34. __ On relatively disjoint families of measures, with some applications to Banach space theory, Studia Math. 37 (1970), 13-36. MR 42 \#5015. 
35. Z. Semadeni, Banach spaces of continuous functions, Vol. I, Monografie Mat., Tom 55, PWN, Warsaw, 1971. MR 45 \# 5730.

36. R. J. Whitley, Some $P_{\lambda}$ Banach spaces, Conf. on Projections, Clemson, South Carolina, 1968.

37. J. Wolfe, Injective Banach spaces of type $C(T)$, Thesis, Univ. of California, Berkeley, 1971.

38. __ Injective Banach spaces of type $C(T)$, Israel J. Math. 18 (1974), 133-140.

Department of Mathematics, Oklahoma State University, Stillwater, Oklahoma 74074 